This item was submitted to Loughborough's Research Repository by the author.

Items in Figshare are protected by copyright, with all rights reserved, unless otherwise indicated.

\title{
Exploiting constructive interference for simultaneous wireless information and power transfer in multiuser downlink systems
}

PLEASE CITE THE PUBLISHED VERSION

http://dx.doi.org/10.1109/JSAC.2016.2551618

PUBLISHER

(C) IEEE

VERSION

AM (Accepted Manuscript)

LICENCE

CC BY-NC-ND 4.0

\section{REPOSITORY RECORD}

Timotheou, Stelios, Gan Zheng, Christos Masouros, and loannis Krikidis. 2019. "Exploiting Constructive Interference for Simultaneous Wireless Information and Power Transfer in Multiuser Downlink Systems". figshare. https://hdl.handle.net/2134/20803. 


\title{
Exploiting constructive interference for simultaneous wireless information and power transfer in multiuser downlink systems
}

\author{
Stelios Timotheou, Member, IEEE, Gan Zheng, Senior Member, IEEE, Christos Masouros, Senior Member, IEEE, \\ and Ioannis Krikidis, Senior Member, IEEE
}

\begin{abstract}
In this paper we propose a power-efficient approach for information and energy transfer in multiple-input singleoutput downlink systems. By means of data-aided precoding, we exploit the constructive part of interference for both information decoding and wireless power transfer. Rather than suppressing interference as in conventional schemes, we take advantage of constructive interference among users, inherent in the downlink, as a source of both useful information signal energy and electrical wireless energy. Specifically, we propose a new precoding design that minimizes the transmit power while guaranteeing the quality of service (QoS) and energy harvesting constraints for generic phase shift keying modulated signals. The QoS constraints are modified to accommodate constructive interference, based on the constructive regions in the signal constellation.

Although the resulting problem is nonconvex, several methods are developed for its solution. First we derive necessary and sufficient conditions for the feasibility of the considered problem. Then we propose second-order cone programming and semidefinite programming algorithms with polynomial complexity that provide upper and lower bounds to the optimal solution and establish the asymptotic optimality of these algorithms when the modulation order and SINR threshold tend to infinity. A practical iterative algorithm is also proposed based on successive linear approximation of the non-convex terms yielding excellent results. More complex algorithms are also proposed to provide tight upper and lower bounds for benchmarking purposes. Simulation results show significant power savings with the proposed data-aided precoding approach compared to the conventional precoding scheme.
\end{abstract}

Keywords: SWIPT, Constructive interference, Beamforming, Power splitting, MISO channel, Optimization.

\section{INTRODUCTION}

Recently simultaneous wireless information and power transfer (SWIPT) via the radio frequency energy harvesting (EH) technology has emerged as a new solution for sustainable wireless network operation. In a multiuser scenario,

S. Timotheou and I. Krikidis are with the KIOS Research Center for Intelligent Systems and Networks, University of Cyprus, Cyprus (E-mail: \{timotheou.stelios, krikidis\}@ucy.ac.cy).

G. Zheng is with the Wolfson School of Mechanical, Electrical and Manufacturing Engineering, Loughborough University, UK (E-mail: eegzheng@gmail.com).

C. Masouros is with the Department of Electronic and Electrical Engineering, University College London, WC1E 7JE London, UK (E-mail: chris.masouros@ieee.org).

Part of this work was supported by the Royal Academy of Engineering, UK and the Engineering and Physical Sciences Research Council (EPSRC) project EP/M014150/1

Part of this work was supported by the Research Promotion Foundation, Cyprus, under the Project FUPLEX with Pr. No. CY-IL/0114/02.

A preliminary version of this work has appeared in ICC 2015, [1]. interference signals provide a source for energy harvesting. On the other hand, there have been some efforts in exploiting constructive interference (CI) to improve the users' quality-ofservice (QoS) and save the transmit power. This paper aims to exploit both data and channel state information (CSI) at the transmitter to facilitate the information and energy transfer in a multiple-input single-output (MISO) broadcast channel. Below we give a brief literature review on simultaneous wireless information and power transfer and data-aided precoding.

\section{Simultaneous Wireless and Information Power Transfer (SWIPT)}

The fundamental concept of simultaneous wireless transmission of energy and information is introduced in [2] from an information theoretic standpoint. In [3] the authors discuss two practical receiver structures for simultaneous wireless information and power transfer termed as "time switching" (TS) and "power splitting" (PS), to separate the received signal for decoding information and harvesting energy, respectively. Furthermore, multi-antenna technology can greatly facilitate the information and power transfer. The optimal precoding design for energy and information transfer in a MISO broadcast channel is studied in [4]. The achievable information and energy transmission trade-offs are investigated in [5] for a 2-user multiple-input multiple-output (MIMO) interference channel (IC), based on different combinations of receiver modes. Joint information and power transfer is studied for a general K-user MISO IC in [6] based on PS receivers where semidefinite relaxation is used to solve the precoding design. A more efficient and decentralized secondorder cone programming (SOCP) relaxation is used in [7]. Energy-efficient wireless power transfer is studied in singleantenna frequency division multiple access systems [8] and multicell coordinated precoding in [9], in which Lagrangian optimization and semidefinite relaxation are used to solve the nonconvex problem.

\section{Beamforming Optimization}

Regarding the precoding design for a broadcast channel, conventional precoding involves a statistical view of interference and focuses on either maximising the QoS - most commonly signal to interference-plus-noise ratio (SINR) - and minimizing interference subject to a transmit power, or minimizing the transmit power subject to QoS constraints. Accordingly a number of optimization problems have been studied 
for broadcast channels in [10], where convex formulations of such nature were proposed. Recent works have focused on the utility maximization in MIMO interfering broadcast channels [12], [13] and full-duplex radios [14]. For the cases of CSI errors, robust versions of these optimization problems have been studied in [15] - [20]. In [15], a robust max-min approach was developed for a single-user MIMO system based on convex optimization. Later in [11], the robust transmission schemes to maximize the compound capacity for single and multiuser rank-one Ricean MIMO channels were addressed, based on the uncertainty set in [16]. Robust beamforming for multiuser MISO downlink channels with individual QoS constraints under an imperfect channel covariance matrix was studied in [10], [17]. Recently in [18], the optimal power allocation over fixed beamforming vectors was obtained in the presence of errors in CSI matrices. Most recently, efficient numerical solutions to find conservative robust beamforming for multiuser MISO systems with mean-square-error (MSE) and SINR constraints and different bounded CSI errors have been developed in [19], [20]. Moreover, SINR balancing optimizations have been proposed in [21] where the minimum achievable SINR is maximized, subject to a total transmit power constraint.

\section{Data-Aided Beamforming for Interference Exploitation}

A newer branch of the downlink beamforming optimization literature, offers an alternative view of the interference, where as opposed to the above statistical approach, interference is treated on an instantaneous basis, by symbol-level precoding. The relevant works focus on exploiting the constructive superposition of useful and interfering signals, to utilise interfering signals as first explored for closed-form precoders [22]- [25]. In [26] a symbol-level precoding is introduced where the conventional optimization constraints are adapted to accommodate constructive interference for phase shift keying modulation (PSK). Further work in [27] focuses on a more relaxed optimization where the optimization constraints are designed based on the constructive interference regions in the PSK constellation, first characterised in [28]. More recent work has extended the above downlink beamforming optimization to quadrature amplitude modulation (QAM) constellations [24], [29].

\section{Our Approach}

In this work, we explore the adaptation of conventional SWIPT beamforming [2]- [6] as per the above symbol level precoding, to exploit an interfering signal as both a source of useful electrical power for $\mathrm{EH}$ and information-driven signal power for the exploitation of constructive interference. Explicitly, we aim to optimize data-aided precoding design in a MISO broadcast channel with PS receivers by exploiting constructive interference as a useful source for both signal and power transfer. We study the problem of transmit power minimization under both SINR and EH constraints.

Our major contribution is that we introduce a new linear precoder design for SWIPT that a) reduces the transmit power for given QoS and EH constraints compared to existing precoders based on the proposed constructive interference regions, and b) as opposed to conventional SWIPT precoders, applies to scenarios with higher number of users than transmit antennas. We re-cast the original optimization problem as a virtual multicast optimization problem in which common messages rather than individual messages are sent to all users using a common beamforming vector. This reformulation greatly simplifies the optimization and facilitates efficient solution.

However, even the simplified problem is still nonconvex. Our explicit contributions to develop algorithmic solutions are listed below:

1) We provide necessary and sufficient conditions to examine the feasibility of the considered problem, which can be checked by solving a linear programming (LP) problem.

2) We formulate polynomial problems that can be solved with SOCP and semi-definite programming (SDP) algorithms which provide lower and upper bounds to the optimal solution.

3) We develop tight upper and lower bounds based on exponential complexity mixed-integer SOCP (MISOCP).

4) We propose a practical iterative algorithm to achieve locally optimal solutions using successive linear approximation for the nonconvex terms.

5) We devise a low-complexity iterative algorithm that provides the optimal solution given a feasible precoding vector.

6) We prove asymptotic optimality of the developed lower and upper bound polynomial complexity algorithms when the SINR threshold and the modulation order tends to infinity.

Our simulation results show that compared to the conventional precoding, the proposed data-aided precoding leads to 4-13 dBW of power saving for all examined cases with one to two orders of magnitude faster execution time.

We note that, while the following analysis focuses on PSK modulation, the above concept and relevant optimizations can be extended to other modulation formats such as QAM as per [29] or by adapting the decision thresholds of the constellation to accommodate for constructive interference [24]. It should be stressed however, that the proposed schemes are most useful in high interference scenarios where low order modulation such as Binary PSK (BPSK) and Quadrature PSK (QPSK) are used in the communication standards to ensure reliability [30]. In addition, constant envelope modulation such as PSK has received particular interest recently with the emergence of large scale MIMO systems [31]. All the above motivate our focus on PSK constellations. Finally, we note that, in line with the relevant literature we assume a time division duplex (TDD) transmission here, where the base station directly estimates the downlink channel using uplink pilot symbols and uplinkdownlink channel reciprocity.

The rest of the paper is organized as follows. Section II introduces the system model and provides a review of conventional precoding design, while Section III formulates the considered optimization problem based on CI precoding. Sections IV and V develop several polynomial complexity algorithms with different performance and execution time 
characteristics to provide upper and lower bound solutions, respectively. Section VI introduces exponential complexity algorithms that yield tight upper and lower bounds to the optimal solution for comparison purposes. Section VII theoretically investigates the feasibility of the considered problem and establishes asymptotic optimality results for the proposed polynomial complexity algorithms. Section VIII illustrates the numerical performance of the developed algorithms compared to optimality and conventional precoding design. Finally, Section IX concludes the paper.

Notation: We use the upper case boldface letters for matrices and lower case boldface letters for vectors. $(\cdot)^{*}$ and $(\cdot)^{T}$ denote the conjugate and transpose, respectively. $\|\cdot\|$ stands for the Frobenius norm. The Rayleigh quotient of matrix $\mathbf{S}$ is defined as $\mathbf{x}^{H} \mathbf{S x} /\left(\mathbf{x}^{H} \mathbf{x}\right) \cdot \lambda_{\max }(\mathbf{S})$ denotes the maximum eigenvalue of matrix $\mathbf{S}$. A complex Gaussian random vector variable $\mathbf{z}$ with mean $\boldsymbol{\mu}$ and variance variance $\boldsymbol{\Sigma}$ is represented as $\mathbf{z} \sim$ $\mathcal{C N}(\boldsymbol{\mu}, \boldsymbol{\Sigma})$. A uniform random variable in the range $[a, b]$ is denoted by $z \sim U(a, b) . \mathbb{E}\{\cdot\}$ denotes the expectation. $\operatorname{Re}(x)$ and $\operatorname{Im}(x)$ denote the real part and imaginary part of a complex number $x \in \mathbb{C}$, respectively.

\section{System Model And Conventional Solution}

\section{A. System Model}

Consider a MISO broadcast channel where an $N$-antenna base station (BS) transmits both signals and energy to $K$ single-antenna users. For user $i$, its channel vector, precoding vector, received noise, data, SINR and $\mathrm{EH}$ constraints are denoted as $\mathbf{h}_{i}^{T}, \mathbf{t}_{i}, n_{i}, d_{i}, \Gamma_{i}, E_{i}$, respectively. The PSK modulated symbol can be expressed as $d_{i}=d e^{j \phi_{i}}$ where $d$ denotes the constant amplitude and $\phi_{i}$ is the phase. Without loss of generality, we assume $d=1$. The average transmit power is

$$
P_{T}=\mathbb{E}\left\{\left\|\sum_{k=1}^{K} \mathbf{t}_{k} d_{k}\right\|^{2}\right\} .
$$

$n_{i} \sim \mathcal{C N}\left(0, N_{0}\right)$ is the additive white Gaussian noise (AWGN). All wireless links exhibit independent frequency non-selective block fading. The received signal at user $i$ is

$$
y_{i}=\mathbf{h}_{i}^{T} \sum_{k=1}^{K} \mathbf{t}_{k} d_{k}+n_{i} .
$$

To decode the information and harvest RF energy at the receiver side, the practical PS technique [3] is used. Specifically, the receiver splits the RF signal into two parts: one for information decoding and the other for energy harvesting, with relative power of $\rho_{i}$ and $1-\rho_{i}$, respectively.

The signal for information decoding is expressed as

$$
\begin{aligned}
\tilde{y}_{i} & =\sqrt{\rho_{i}} y_{i}+\tilde{n}_{i} \\
& =\sqrt{\rho_{i}} \mathbf{h}_{i}^{T} \sum_{k=1}^{K} \mathbf{t}_{k} d_{k}+\sqrt{\rho_{i}} n_{i}+\tilde{n}_{i},
\end{aligned}
$$

where $\tilde{n}_{i} \sim \mathcal{C N}\left(0, N_{C}\right)$ is the complex AWGN introduced in the RF to baseband conversion in the decoding process, which is independent of $n_{i}$.
The signal for energy harvesting is

$$
\bar{y}_{i}=\sqrt{1-\rho_{i}} y_{i}=\sqrt{1-\rho_{i}}\left(\mathbf{h}_{i}^{T} \sum_{k=1}^{K} \mathbf{t}_{k} d_{k}+n_{i}\right)
$$

with average power

$$
P_{i}=\left(1-\rho_{i}\right) \mathbb{E}\left\{\left|\mathbf{h}_{i}^{T} \sum_{k=1}^{K} \mathbf{t}_{k} d_{k}+n_{i}\right|^{2}\right\} .
$$

The problem of interest is to minimize the total transmit power $P_{T}$ in (1) subject to QoS (i.e., SINR) constraints $\left\{\Gamma_{i}\right\}$ and energy harvesting constraints $\left\{E_{i}\right\}$, respectively. This will be achieved by optimizing beamforming design, power allocation and splitting, by exploiting the CI concept.

In the following, we first review the conventional precoding design then we introduce the proposed approach based on constructive interference.

\section{B. Review: Conventional Precoding}

In conventional MISO downlink precoding, users' data are independent of each other, i.e., $\mathbb{E}\left(d_{i}^{*} d_{j}\right)=0, \forall j \neq i$. In this case, the transmit power in (1) becomes

$$
P_{T}=\sum_{i=1}^{K}\left\|\mathbf{t}_{i}\right\|^{2}
$$

Based on the signal model (3) for information decoding, the received SINR for user $i$ is given by

$$
\Gamma_{i}^{c o n}=\frac{\left|\mathbf{h}_{i}^{T} \mathbf{t}_{i}\right|^{2}}{\sum_{j=1, j \neq i}^{K}\left|\mathbf{h}_{i}^{T} \mathbf{t}_{j}\right|^{2}+N_{0}+\frac{N_{C}}{\rho_{i}}} .
$$

The harvested energy can be derived from (5) as

$$
P_{i}^{\text {con }}=\left(1-\rho_{i}\right)\left(\sum_{k=1}^{K}\left|\mathbf{h}_{i}^{T} \mathbf{t}_{k}\right|^{2}+N_{0}\right) .
$$

Consequently, the power minimization problem with both QoS and $\mathrm{EH}$ constraints can be formulated as

$$
\begin{aligned}
\min _{\left\{\mathbf{t}_{i}, \rho_{i}\right\}} & \sum_{i=1}^{K}\left\|\mathbf{t}_{i}\right\|^{2} \\
\text { s.t. } & \Gamma_{i}^{c o n} \geq \Gamma_{i}, \quad P_{i}^{c o n} \geq E_{i}, 0<\rho_{i}<1, \forall i .
\end{aligned}
$$

It is easy to see that formulation (8) is non-convex and hence challenging to solve. In our previous work [6], we have used semidefinite programming relaxation to tackle it and we show that the relaxation is tight for 2-user and 3-user MISO IC. This result is extended in [4], showing that the SDP relaxation is tight for the general MISO downlink case $(K \in \mathbb{N})$.

\section{Proposed Precoding with constructive INTERFERENCE}

\section{A. Problem Formulation}

Traditional precoding techniques refer to Gaussian information signals and treat users' data as totally random and independent information streams, and the interference between them as harmful. For the PSK signalling considered, however, 


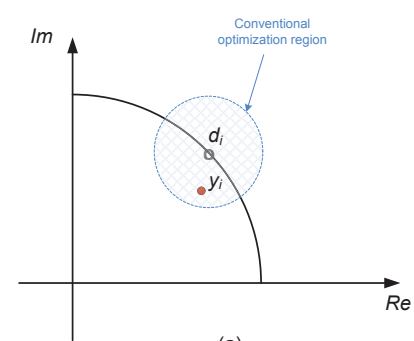

(a)

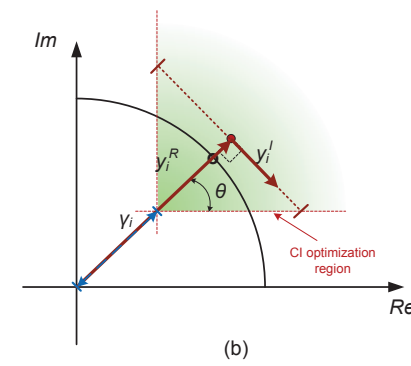

(b)
Fig. 1. Illustration of constructive interference for information decoding, QPSK example.

interference can be constructive to the signal's detection on an instantaneous basis when it shifts the received constellation point away from the decision thresholds in the constellation [22]- [26]. Therefore, in this context one user's data do not always generate harmful interference to others. With the knowledge of both the instantaneous CSI and the data symbols at the BS, the received interference can be classified to be constructive or destructive. In brief, while destructive interference deteriorates performance, CI moves the received symbols away from the decision thresholds of the constellation and thus improves the detection. We refer the readers to [22]-[24] for further details. The main idea of the proposed precoding is to exploit the CI for both information decoding and energy harvesting.

The received signal at user $i$ in (2) can be rewritten as

$$
\begin{aligned}
y_{i} & =\mathbf{h}_{i}^{T} \sum_{k=1}^{K} \mathbf{t}_{k} d_{k}+n_{i} \\
& =\mathbf{h}_{i}^{T} \sum_{k=1}^{K} \mathbf{t}_{k} e^{j\left(\phi_{k}-\phi_{i}\right)} d_{i}+n_{i} .
\end{aligned}
$$

The information decoding part can be written as

$$
\sqrt{\rho_{i}} y_{i}+\tilde{n}_{i}=\sqrt{\rho_{i}} \mathbf{h}_{i}^{T} \sum_{k=1}^{K} \mathbf{t}_{k} e^{j\left(\phi_{k}-\phi_{i}\right)} d_{i}+\sqrt{\rho_{i}} n_{i}+\tilde{n}_{i}
$$

We illustrate the derivation of the SINR constraint for the example of QPSK in Fig. 1. Here, Fig. 1(a) represents the conventional optimization region and Fig. 1(b) shows the proposed optimization region. We have used the definitions $y_{i}^{R}=\operatorname{Re}\left(\mathbf{h}_{i}^{T} \sum_{k=1}^{K} \mathbf{t}_{k} e^{j\left(\phi_{k}-\phi_{i}\right)}\right), y_{i}^{I}=$ $\operatorname{Im}\left(\mathbf{h}_{i}^{T} \sum_{k=1}^{K} \mathbf{t}_{k} e^{j\left(\phi_{k}-\phi_{i}\right)}\right)$ and $\gamma_{i}=\sqrt{\Gamma_{i}\left(N_{0}+\frac{N_{C}}{\rho_{i}}\right)}$.

By means of their definition, $y_{i}^{R}$ and $y_{i}^{I}$ essentially shift the observation of received symbol onto the axis from the origin of the constellation diagram to the constellation symbol of interest. Clearly, $y_{i}^{R}$ provides a measure of the amplification of the received constellation point along the axis of the nominal constellation point due to constructive interference, and $y_{i}^{I}$ provides a measure of the angle shift from the original constellation point, i.e. the deviation from the axis of the nominal constellation point with phase $\phi_{i}$. The reader is referred to [27] for further details where this concept is explained in the context of downlink precoding.
In conventional precoding optimization, $y_{i}^{R}$ and $y_{i}^{I}$ are constrained such that the received symbol is contained within a circle (denoted by the dashed circle in Fig. 1(a)) around the nominal constellation point, so that the interference caused by the other symbols is limited. In contrast to this, the concept of $\mathrm{CI}$ is exploited to allow a relaxation of $y_{i}^{R}$ and $y_{i}^{I}$ for all transmit symbols, under the condition that the interference caused is constructive, lying in the green shaded sector in the diagram [23]. It can be seen that $y_{i}^{R}$ and $y_{i}^{I}$ are allowed to grow infinitely, as long as their ratio is kept such that the received symbol is contained within the constructive area of the constellation, i.e., the distances from the decision thresholds, as set by the SNR constraints $\gamma_{i}$, are not violated. It can be seen that the angle of interference need not be strictly aligned with the angle of the useful signal, as long as it falls within the constructive area of the constellation. For a given modulation order $M$ the maximum angle shift in the CI area is given by $\theta=\pi / M$. By using basic geometry we arrive at the SINR constraint expressed as [27]

$$
\left|y_{i}^{I}\right| \leq\left(y_{i}^{R}-\gamma_{i}\right) \tan \theta
$$

which is expanded to

$$
\begin{aligned}
& \left|\operatorname{Im}\left(\mathbf{h}_{i}^{T} \sum_{k=1}^{K} \mathbf{t}_{k} e^{j\left(\phi_{k}-\phi_{i}\right)}\right)\right| \leq \\
& \left(\operatorname{Re}\left(\mathbf{h}_{i}^{T} \sum_{k=1}^{K} \mathbf{t}_{k} e^{j\left(\phi_{k}-\phi_{i}\right)}\right)-\sqrt{\Gamma_{i}\left(N_{0}+\frac{N_{C}}{\rho_{i}}\right)}\right) \tan \theta .
\end{aligned}
$$

The harvested energy and the total transmit power can be derived based on (1) and (5), respectively, as $P_{i}=$ $\left|\mathbf{h}_{i}^{T} \sum_{k=1}^{K} \mathbf{t}_{k} e^{j\left(\phi_{k}-\phi_{i}\right)}\right|^{2}$ and $P_{T}=\left\|\sum_{k=1}^{K} \mathbf{t}_{k} e^{j\left(\phi_{k}-\phi_{i}\right)}\right\|^{2}$. Therefore, the power minimization problem subjective to both SINR and EH constraints with the aid of the CI can be formulated as

$$
\begin{aligned}
& \min _{\left\{\mathbf{t}_{i}, \rho_{k}\right\}}\left\|\sum_{k=1}^{K} \mathbf{t}_{k} e^{j\left(\phi_{k}-\phi_{i}\right)}\right\|^{2} \\
& \text { s.t. constraint (12), } \\
& \left|\mathbf{h}_{i}^{T} \sum_{k=1}^{K} \mathbf{t}_{k} e^{j\left(\phi_{k}-\phi_{i}\right)}\right| \geq \sqrt{\frac{E_{i}}{1-\rho_{i}}}, i \in \mathcal{K} \\
& 0<\rho_{i}<1, i \in \mathcal{K} \text {. }
\end{aligned}
$$

By defining $\tilde{\mathbf{h}}_{i}=\mathbf{h}_{i} e^{j\left(\phi_{1}-\phi_{i}\right)}$ and $\mathbf{w} \triangleq \sum_{k=1}^{K} \mathbf{t}_{k} e^{j\left(\phi_{k}-\phi_{1}\right)}$, we can write (13) equivalently as

$$
\begin{aligned}
& \min _{\{\mathbf{w}, \boldsymbol{\rho}\}}\|\mathbf{w}\|^{2} \\
& \text { s.t. }\left|\operatorname{Im}\left(\tilde{\mathbf{h}}_{i}^{T} \mathbf{w}\right)\right| \leq\left(\operatorname{Re}\left(\tilde{\mathbf{h}}_{i}^{T} \mathbf{w}\right)\right. \\
& \left.-\sqrt{\Gamma_{i}\left(N_{0}+\frac{N_{C}}{\rho_{i}}\right)}\right) \tan \theta, i \in \mathcal{K} \\
& \left\|\tilde{\mathbf{h}}_{i}^{T} \mathbf{w}\right\|^{2} \geq \frac{E_{i}}{1-\rho_{i}}, i \in \mathcal{K} \\
& 0<\rho_{i}<1, i \in \mathcal{K} \text {. }
\end{aligned}
$$


Although the reformulation (14) seems a trivial step, it indicates that the original broadcast channel reduces to a virtual multicast channel with common messages to all users [32].

The problem (14) is nontrivial to solve because of the nonconvex constraint $\left|\tilde{\mathbf{h}}_{i}^{T} \mathbf{w}\right|^{2} \geq \frac{E_{i}}{1-\rho_{i}}$. The rest of this paper is devoted to solving the multicast problem (14).

\section{ApProximate SOCP SOlutions}

In this section we develop two algorithms that provide approximate solutions to the considered problem. The first approximates the nonconvex constraints to formulate and solve an SOCP convex problem. The second builds upon the first solution to provide a better solution using successive linear approximation. A brief introduction on SOCP formulations is provided before describing these algorithms.

SOCP problems are convex formulations involving the minimization of a linear function subject to linear and secondorder cone (SOC) constraints [33]. Their standard form is:

$$
\begin{aligned}
\min _{\mathbf{x}} & \mathbf{c}^{T} \mathbf{x} \\
\text { s.t. } & \mathbf{A} \mathbf{x}=\mathbf{b}, \\
& \quad\left\|\mathbf{C}_{i} \mathbf{x}-\mathbf{d}_{i}\right\| \leq \mathbf{e}_{i}^{T} \mathbf{x}+f_{i}, i=1, \ldots, L_{C},
\end{aligned}
$$

Among the constraints that can be modeled using SOCs are the restricted hyperbolic constraints which have the form: $\mathbf{x}^{T} \mathbf{x} \leq$ $y z$, where $\mathbf{x} \in \mathbb{C}^{N \times 1}, y, z \geq 0$. Such a constraint is equivalent to a rotated SOC constraint of the form:

$$
\left\|\left(\begin{array}{c}
2 \mathbf{x} \\
y-z
\end{array}\right)\right\| \leq y+z .
$$

For example, constraint $x^{2} \leq y z, y, z \geq 0$ is equivalent to SOC $\left\|[2 x, y-z]^{T}\right\| \leq y+z$. Also, the geometric mean constraint (GMC) $x_{1} \ldots x_{2^{l}} \geq t^{2^{l}}, x_{i} \geq 0, i=1, \ldots, 2^{l}$ is represented by a hierarchy of SOC constraints by defining existing variables as $x_{i} \equiv x_{0, i}$ and new variables $x_{k, i} \geq$ $0, k=1, \ldots, l, i=1, \ldots, 2^{l-k}$ so that GMC is equivalent to [34, p.105]:

layer $k: x_{k-1,2 i-1} x_{k-1,2 i} \geq x_{k, i}^{2}, i=1, \ldots, 2^{l-k}, k=1, \ldots, l$,

$$
x_{l, 1} \geq t .
$$

\section{A. Upper bounding SOCP algorithm}

In this section, an upper bound solution to (14) is derived by approximating the problem using convex SOCP. Towards this direction, we begin by reformulating (14b) for $i \in \mathcal{K}$ using SOCP constraints. If we define

$$
\begin{aligned}
& v_{i}=\left|\operatorname{Im}\left(\tilde{\mathbf{h}}_{i}^{T} \mathbf{w}\right)\right|, \\
& y_{i}^{R}=\operatorname{Re}\left(\tilde{\mathbf{h}}_{i}^{T} \mathbf{w}\right)=\sum_{k=1}^{K} \operatorname{Re}\left(\tilde{h}_{i, k}\right) w_{k}^{R}-\operatorname{Im}\left(\tilde{h}_{i, k}\right) w_{k}^{I}, \\
& y_{i}^{I}=\operatorname{Im}\left(\tilde{\mathbf{h}}_{i}^{T} \mathbf{w}\right)=\sum_{k=1}^{K} \operatorname{Im}\left(\tilde{h}_{i, k}\right) w_{k}^{R}+\operatorname{Re}\left(\tilde{h}_{i, k}\right) w_{k}^{I},
\end{aligned}
$$

then it is true that the absolute term of (14b) can be equivalently represented by two linear constraints as:

$$
y_{i}^{I} \leq v_{i}, \quad-y_{i}^{I} \leq v_{i}, i \in \mathcal{K} .
$$

This is true because on the one hand, constraint (17) forces $v_{i} \geq\left|y_{i}^{I}\right|, i \in \mathcal{K}$, and on the other hand, (14b) forces $v_{i}$ to be as small as possible, which is achieved for $v_{i}=\left|y_{i}^{I}\right|$.

To deal with the square root, the terms in (14b) are rearranged and both sides of the constraint are squared yielding

$$
\left(y_{i}^{R}-v_{i} / \tan \theta\right)^{2} \geq \Gamma_{i}\left(N_{0}+\frac{N_{C}}{\rho_{i}}\right),
$$

which is equivalent to

$$
\begin{aligned}
z_{i}^{+} & =y_{i}^{R}-v_{i} / \tan \theta+\sqrt{\Gamma_{i} N_{0}}, \\
z_{i}^{-} & =y_{i}^{R}-v_{i} / \tan \theta-\sqrt{\Gamma_{i} N_{0}}, \\
z_{i}^{+} z_{i}^{-} & \geq \frac{\Gamma_{i} N_{C}}{\rho_{i}} .
\end{aligned}
$$

From the constraint in (14b), it is easy to see that a solution of (14) satisfies $y_{i}^{R}>v_{i} / \tan \theta \geq 0$, and hence $z_{i}^{+}>0$. Because the right hand side (r.h.s.) of (20) is positive, combined with the fact that $z_{i}^{+}>0$, implies that a solution of (14) satisfies $z_{i}^{+}, z_{i}^{-}>0$; hence, based on the SOCP hierarchy for GMC, (20) is equivalent to:

$$
\begin{aligned}
& z_{i}^{+} z_{i}^{-} \geq r_{1, i}^{2}, \quad \rho_{i} \geq r_{2, i}^{2}, \\
& r_{1, i} r_{2, i} \geq \sqrt{\Gamma_{i} N_{C}}, \\
& z_{i}^{+} \geq 0, z_{i}^{-} \geq 0, r_{1, i} \geq 0, r_{2, i} \geq 0 .
\end{aligned}
$$

To summarize, constraint (14b) is equivalent to (15)-(19) and (21)-(23).

Constraint (14c), is not convex due to the term $\left\|\tilde{\mathbf{h}}_{i}^{T} \mathbf{w}\right\|^{2}=$ $\left(y_{i}^{R}\right)^{2}+\left(y_{i}^{I}\right)^{2}, i \in \mathcal{K}$; nonetheless, it can be convexified by eliminating the real or imaginary part. Eliminating the imaginary part is better because $y_{i}^{R} \geq\left|v_{i}\right| / \tan \theta+\sqrt{\Gamma_{i} N_{0}}$ yielding the constraint:

$$
\left(y_{i}^{R}\right)^{2} \geq \frac{E_{i}}{1-\rho_{i}},
$$

which is similar to (20), and hence can be reformulated into SOCP. Hence, the approximate SOCP formulation is:

$$
\min _{\left\{\mathbf{w}, \boldsymbol{\rho}, \mathbf{z}_{i}^{ \pm}, \mathbf{r}_{1}, \mathbf{r}_{2}, \mathbf{r}_{3}, \mathbf{y}^{R}, \mathbf{y}^{I}, \mathbf{v}\right\}}\|\mathbf{w}\|^{2}
$$

s.t. Constraints (15)-(19), (21)-(23), (14d),

$$
\begin{aligned}
& 1-\rho_{i} \geq r_{3, i}^{2}, i \in \mathcal{K}, \\
& r_{3, i} y_{i}^{R} \geq \sqrt{E_{i}}, i \in \mathcal{K}, \\
& r_{3, i} \geq 0, y_{i}^{R} \geq 0 i \in \mathcal{K} .
\end{aligned}
$$

Problem (25) provides an upper bound to the solution of (14), as its solution is always feasible for the latter since $\left(y_{i}^{R}\right)^{2}+\left(y_{i}^{I}\right)^{2} \geq\left(y_{i}^{R}\right)^{2}$. Note that if $y_{i}^{I}=0, i \in \mathcal{K}$, then this formulation provides an optimal solution.

\section{B. Successive linear approximation algorithm}

In this section, we propose an iterative approach to tackle the nonconvex constraint (14b) $\left(y_{i}^{R}\right)^{2}+\left(y_{i}^{I}\right)^{2} \geq \frac{E_{i}}{1-\rho_{i}}$, using successive linear approximation (SLA). To illustrate the idea, we define the quadratic function $f(v) \triangleq v^{2}$, which has the same form with the nonconvex terms $\left(y_{i}^{R}\right)^{2}$ and $\left(y_{i}^{I}\right)^{2}, i \in \mathcal{K}$. 


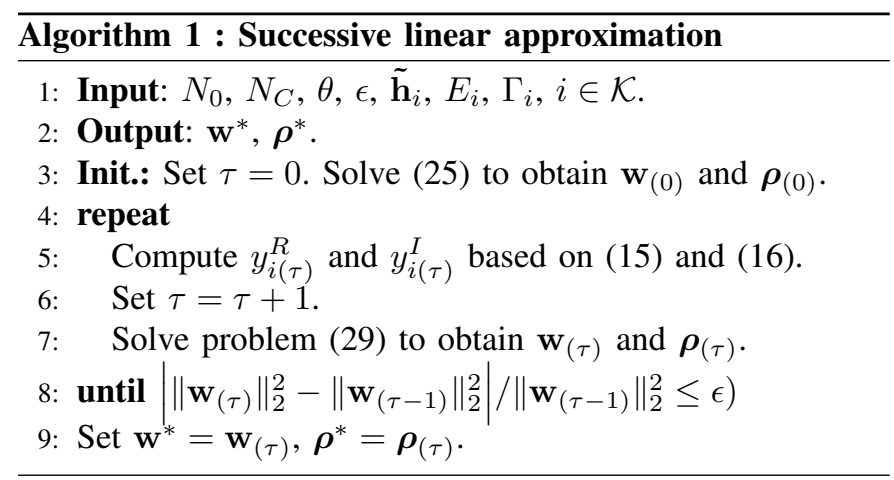

The linear approximation of $f(v)$ around the point $p$ can be expressed as

$$
\begin{aligned}
f(v) & \approx f(p)+\left(\left.\frac{d f(v)}{d v}\right|_{v=p}\right)(v-p) \\
& =p^{2}+2 p(v-p)
\end{aligned}
$$

It is important to note that the first order approximation $p^{2}+$ $2(v-p)$ always approximates the quadratic function $v^{2}$ from below such that $v^{2} \geq p^{2}+2(v-p)$, for all $v, p$.

Let $y_{i(\tau)}^{R}$ and $y_{i(\tau)}^{I}$ denote the values of variables $y_{i}^{R}$ and $y_{i}^{I}$ obtained using $\mathbf{w}_{(\tau)}$ at the $\tau$-th iteration of the algorithm. Performing linear approximation of the nonconvex constraints yields

$$
\omega_{i} \geq E_{i} /\left(1-\rho_{i}\right), i \in \mathcal{K}
$$

where $\omega_{i}$, for $i \in \mathcal{K}$ is given by

$$
\omega_{i}=\left(y_{i(\tau)}^{R}\right)^{2}+2\left(y_{i}^{R}-y_{i(\tau)}^{R}\right)+\left(y_{i(\tau)}^{I}\right)^{2}+2\left(y_{i}^{I}-y_{i(\tau)}^{I}\right) .
$$

Hence, the problem that needs to be solved during the $\tau+1$ iteration is given by:

$$
\begin{aligned}
& \min _{\left\{\mathbf{w}, \boldsymbol{\rho}, \mathbf{z}_{i}^{ \pm}, \mathbf{r}_{1}, \mathbf{r}_{2}, \boldsymbol{\rho}_{2}, \mathbf{y}^{R}, \mathbf{y}^{I}, \mathbf{v}, \boldsymbol{\omega}\right\}}\|\mathbf{w}\|^{2} \\
& \text { s.t. Constraints (15)-(19), (21)-(23), (14d),(28) } \\
& \rho_{2 i}=1-\rho_{i}, i \in \mathcal{K} \\
& \omega_{i} \rho_{2 i} \geq E_{i}, \quad u_{i} \geq 0, \quad i \in \mathcal{K} .
\end{aligned}
$$

SLA is outlined in Algorithm 1. The algorithm starts by solving problem (25) to obtain an initially feasible solution. Then, an iterative procedure is followed which involves linear approximation of the nonconvex terms upon derivation of $y_{i(\tau)}^{R}$ and $y_{i(\tau)}^{I}$, followed by the solution of problem (29) to obtain a new approximate solution to the initial problem. The procedure is repeated until the relative difference of the objective function between two successive iterations is below a threshold $\epsilon$. It is important to note that $\left(y_{i}^{R}\right)^{2}+\left(y_{i}^{I}\right)^{2} \geq \omega_{i}$ is always true, which implies that solving (29) always provides a feasible solution to (14). SLA is a widely used procedure in signal processing for communications that has been proven to converge to a local optimum [35]. Algorithm 1 starts from the solution of (25) and progressively improves so that its solution is at least as good as the one of (25). Hence, there is a computational complexity/solution quality tradeoff between formulation (25) and Algorithm 1 as the former has lower computational complexity, while the latter yields results closer to optimality.

\section{Improving Feasible Solutions}

In sections IV-A and IV-B procedures have been developed to obtain feasible solutions to problem (14) using SOCP. This section proposes a low-complexity algorithm to improve upon a feasible solution $\hat{\mathbf{w}}, \hat{\boldsymbol{\rho}}$, i.e. to obtain a new solution with lower total transmit power. The key idea is to attempt to find an optimal value for variable $0<\delta<1$ such that $\tilde{\mathbf{w}}=\delta \hat{\mathbf{w}}$ and $\tilde{\boldsymbol{\rho}}$, is a feasible solution to problem (14). Replacing $\mathbf{w}$ with $\delta \hat{\mathbf{w}}$ in formulation (14) yields:

$$
\begin{aligned}
& \min _{\{\delta, \boldsymbol{\rho}\}} \delta^{2}\|\hat{\mathbf{w}}\|^{2} \\
& \text { s.t. } \delta\left|\operatorname{Im}\left(\tilde{\mathbf{h}}_{i}^{T} \hat{\mathbf{w}}\right)\right| \leq\left(\delta \operatorname{Re}\left(\tilde{\mathbf{h}}_{i}^{T} \hat{\mathbf{w}}\right)\right. \\
& \left.-\sqrt{\Gamma_{i}\left(N_{0}+\frac{N_{C}}{\rho_{i}}\right)}\right) \tan \theta, i \in \mathcal{K}, \\
& \delta^{2}\left\|\tilde{\mathbf{h}}_{i}^{T} \hat{\mathbf{w}}\right\|^{2} \geq \frac{E_{i}}{1-\rho_{i}}, i \in \mathcal{K} \\
& 0<\rho_{i}<1, i \in \mathcal{K} .
\end{aligned}
$$

Notice that the objective function of (30) is unimodal and involves only variable $\delta$. Hence, one solution approach is to apply the bisection method, which examines whether problem (30) is feasible for a specific $\delta$ and appropriately increases or decreases $\delta$. Notice also that for fixed $\delta$ the problem can be decomposed into $K$ separable 1-D subproblems involving variables $\rho_{i}$. Rearranging the terms in (30b) and (30c) for subproblem $i$ yields:

$$
\begin{aligned}
& \rho_{i} \geq\left(\Gamma_{i} N_{C}\right) /\left(\eta_{1 i} \delta^{2}-\Gamma_{i} N_{0}\right)=\rho_{i}^{L B}, \\
& \rho_{i} \leq 1-E_{i} /\left(\eta_{2 i} \delta^{2}\right)=\rho_{i}^{U B},
\end{aligned}
$$

where

$$
\begin{aligned}
\eta_{1 i} & =\left(\operatorname{Re}\left(\tilde{\mathbf{h}}_{i}^{T} \hat{\mathbf{w}}\right)-\left|\operatorname{Im}\left(\tilde{\mathbf{h}}_{i}^{T} \hat{\mathbf{w}}\right)\right| / \tan \theta\right)^{2} \\
\eta_{2 i} & =\left\|\tilde{\mathbf{h}}_{i}^{T} \hat{\mathbf{w}}\right\|^{2}
\end{aligned}
$$

Feasibility of inequalities (31) and (32) is achieved when $0<\rho_{i}^{L B}<\rho_{i}^{U B}<1$. If feasibility is true for all $i \in \mathcal{K}$, then solution $\delta \hat{\mathbf{w}}$ is feasible for problem (30) otherwise the problem is deemed infeasible for the particular value of $\delta$.

Each iteration of the bisection method is $O(K)$ and since convergence through bisection is achieved in approximately $\log _{2}(1 / \epsilon)$ iterations, where $\epsilon$ is an optimality tolerance, the total complexity of the algorithm is $O\left(K \log _{2}(1 / \epsilon)\right)$ which is significantly smaller than the complexity of SOCP or SDP algorithms.

\section{LOWER BOUNDING FORMULATIONS}

In this section two formulations are developed that provide lower bounds to the optimal solution of (14). These include an SOCP formulation and an SDP one that builds upon the SOCP formulation to provide tighter bounds. Developing lower bounding formulations is important because they provide an indication of how far a solution is from the optimal. 


\section{A. SOCP lower bounding formulation}

In order to obtain an SOCP LB solution, we need to approximate from below the non-convex term in $(14 \mathrm{c})$. For this reason, we consider that $y_{i}^{R} \geq v_{i} / \tan \theta$, which implies that $\left(y_{i}^{R}\right)^{2}+y_{i}^{R} v_{i} \tan \theta \leq\left(y_{i}^{R}\right)^{2}+\left(y_{i}^{I}\right)^{2}$. Because $\left(y_{i}^{R}\right)^{2}+y_{i}^{R} v_{i} \tan \theta$ can be expressed as the product of two positive linear terms, i.e., $y_{i}^{R}\left(y_{i}^{R}+v_{i} \tan \theta\right)$, the resulting constraint can be expressed into a convex SOCP form similar to $(20)$, yielding the formulation:

$$
\min _{\left\{\mathbf{w}, \boldsymbol{\rho}, \mathbf{z}_{i}^{ \pm}, \mathbf{r}_{1}, \mathbf{r}_{2}, \mathbf{r}_{3}, \mathbf{r}_{4}, \mathbf{u}, \mathbf{y}^{R}, \mathbf{y}^{I}, \mathbf{v}\right\}}\|\mathbf{w}\|^{2}
$$

s.t. Constraints (15)-(19), (21)-(23), (14d),

$$
\begin{aligned}
& 1-\rho_{i} \geq r_{3, i}^{2}, i \in \mathcal{K}, \\
& u_{i}=y_{i}^{R}+v_{i} \tan \theta, i \in \mathcal{K}, \\
& y_{i}^{R} u_{i} \geq r_{4, i}^{2}, i \in \mathcal{K}, \\
& r_{3, i} r_{4, i} \geq \sqrt{E_{i}}, i \in \mathcal{K}, \\
& r_{3, i} \geq 0, r_{4, i} \geq 0, u_{i} \geq 0, i \in \mathcal{K} .
\end{aligned}
$$

\section{B. SDP lower bound formulation}

The SDP relaxation for the nonconvex constraint has been proven very useful in providing tight and in some cases optimal results [4], [7]. The difficulty in developing the SDP relaxation for this problem stems from the square root and fractional terms of constraints (14b) and (14c), respectively. Nevertheless, we have already transformed these constraints into equivalent SOCP constraints. It is true that SOCP constraints of the form $x y \geq z^{2}, x, y \geq 0$ can be expressed into the following SDP form:

$$
x y \geq z^{2}, x, y \geq 0, \Leftrightarrow\left[\begin{array}{ll}
x & z \\
z & y
\end{array}\right] \geq 0 .
$$

Utilizing this constraint, the SDP lower bound relaxation of (14) is obtained by defining $\mathbf{W}=\mathbf{w} \mathbf{w}^{T}$ and relaxing this equality with $\mathbf{W} \succeq \mathbf{w w}^{T}$ :

$$
\begin{aligned}
& \min _{\left\{\mathbf{W}, \mathbf{w}, \boldsymbol{\rho}, \mathbf{y}^{R}, \mathbf{y}^{I}, \mathbf{v}, \mathbf{r}_{1}, \mathbf{r}_{2}\right\}} \operatorname{trace} \operatorname{Re}(\mathbf{W}) \\
& \text { s.t.Constraints (15) - (17), } \\
& {\left[\begin{array}{ll}
y_{i}^{R}-\frac{v_{i}}{\tan \theta}-\sqrt{\Gamma_{i} N_{0}} & r_{1, i} \\
r_{1, i} & y_{i}^{R}-\frac{v_{i}}{\tan \theta}+\sqrt{\overline{\Gamma_{i} N_{0}}}
\end{array}\right] \succeq 0, i \in \mathcal{K}} \\
& {\left[\begin{array}{ll}
\rho_{i} & r_{2, i} \\
r_{2, i} & 1
\end{array}\right] \geq 0, \quad\left[\begin{array}{ll}
r_{1, i} & \left(\Gamma_{i} N_{C}\right)^{\frac{1}{4}} \\
\left(\Gamma_{i} N_{C}\right)^{\frac{1}{4}} & r_{2, i}
\end{array}\right] \succeq 0, i \in \mathcal{K}} \\
& {\left[\begin{array}{ll}
\operatorname{Re}\left(\operatorname{trace}\left(\left(\tilde{\mathbf{h}}_{i}^{*} \tilde{\mathbf{h}}_{i}^{T}\right) \mathbf{W}\right)\right) & \left(\Gamma_{i} N_{C}\right)^{1 / 4} \\
\left(\Gamma_{i} N_{C}\right)^{1 / 4} & 1-\rho_{i}
\end{array}\right] \succeq 0, i \in \mathcal{K}} \\
& {\left[\begin{array}{ll}
\mathbf{W} & \mathbf{w} \\
\mathbf{w}^{T} & 1
\end{array}\right] \succeq 0, \quad \mathbf{W} \succeq 0,} \\
& 0<\rho_{i}<1, r_{1, i} \geq 0, r_{2, i} \geq 0, i \in \mathcal{K} .
\end{aligned}
$$

Rewriting constraints $(35 \mathrm{c})-(35 \mathrm{~g})$ that provide a lower bound on the EH constraint into an equivalent SDP form and combining them with formulation (37) yields

$$
\min _{\left\{\mathbf{W}, \mathbf{w}, \boldsymbol{\rho}, \mathbf{y}^{R}, \mathbf{y}^{I}, \mathbf{v}, \mathbf{r}_{l}, l=1, \ldots, 4\right\}} \operatorname{trace} \operatorname{Re}(\mathbf{W})
$$

s.t. Constraints (15)-(17), (37c)-(37g),

$$
\begin{aligned}
& {\left[\begin{array}{ll}
1-\rho_{i} & r_{3, i} \\
r_{3, i} & 1
\end{array}\right] \succeq 0, i \in \mathcal{K}} \\
& {\left[\begin{array}{ll}
y_{i}^{R} & r_{4, i} \\
r_{4, i} & y_{i}^{R}+v_{i} \tan \theta
\end{array}\right] \succeq 0, i \in \mathcal{K}} \\
& {\left[\begin{array}{ll}
r_{3, i} & \left(E_{i}\right)^{1 / 4} \\
\left(E_{i}\right)^{1 / 4} & r_{4, i}
\end{array}\right] \succeq 0, i \in \mathcal{K}} \\
& r_{3, i} \geq 0, r_{4, i} \geq 0, i \in \mathcal{K} .
\end{aligned}
$$

SDP formulation (38) provides a lower bound to (14); it is optimal if $\operatorname{rank}(\mathbf{W})=1$, in which case it is true that $\mathbf{W}=\mathbf{w w}^{T}$. Note also that (38) is tighter than both (35) and (37), as it combines both lower bounding formulations. Finally, notice that there is a computational complexity/solution quality tradeoff between formulations (35) and (38) as the former has lower computational complexity, while the latter yields tighter lower bounds.

\section{TIGHT MISOCP BOUNDS}

Due to the non-convexity of (14), in Sections IV and V we have proposed several algorithms to obtain polynomial but suboptimal upper and lower bound solutions. To evaluate the performance of these algorithms in terms of optimality, in this section a MISOCP formulation is developed, which provides tight lower and upper bounds to the optimal solution. This is achieved by approximating the non-convex quadratic terms, $\left(y_{i}^{R}\right)^{2}$ and $\left(y_{i}^{I}\right)^{2}$, in (14c) into mixed-integer linear expressions using piecewise linear approximation (PLA) functions. Note that considering an upper (lower) PLA envelope yields a LB (UB) to problem (14), as the resulting PLA function overestimates (underestimates) the values of $\left(y_{i}^{R}\right)^{2}+\left(y_{i}^{I}\right)^{2}$. Naturally, by tightening the PLA of the quadratic terms, better bounds are obtained, but this requires more linear segments resulting in higher computational cost. Note that these approaches have exponential complexity and are only used for benchmarking purposes.

One can easily find the PLA of a quadratic function, by considering a fixed number of points, at integer multiples of a parameter $\kappa$, such that segments are either tangent (lower envelope) or intersect (upper envelope) the quadratic function. Nonetheless, this approach has no control over the approximation error, while the maximum error for different segments varies. For this reason, an approach has been followed that produces segments when needed so that the approximation error does not exceed a predefined value $e_{m}$ [37]. Although this approach may lead to a large number of segments we can adjust $e_{m}$ to achieve the desirable number of segments and at the same time maintain the error less than a constant known value.

To illustrate the PLA procedure, here we only consider a general variable $y$ in place of $y_{i}^{R}$ and $y_{i}^{I}$ and an approximation interval $\left\{y_{L}, y_{U}\right\}$. The approximation procedure, involves two steps for the production of each segment. In UPLA, where an upper envelope needs to be produced, the first step for the derivation of segment $l$, starts from a known intersection point $\left\{y_{l}, y_{l}^{2}\right\}$ (initially it is set $y_{1}=y_{L}$ ) and finds a line $\alpha_{l} y+\beta_{l}$ such that $y^{2}-\left(\alpha_{l} y+\beta_{l}\right) \leq e_{m}$, for $y^{2} \geq \alpha_{l} y+\beta_{l}$. In the second step, a new intersection point is found, such that 


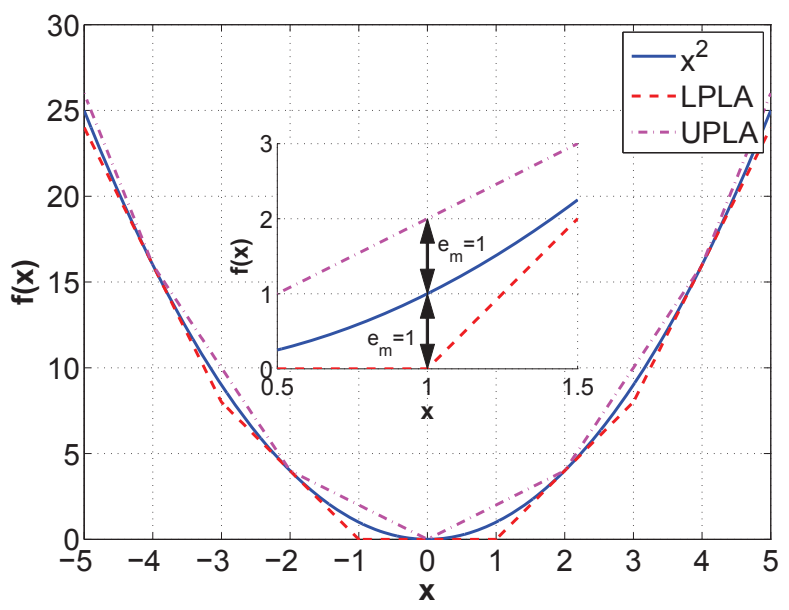

Fig. 2. Illustration of upper and lower bound approximation of function $x^{2}$ for the interval $[-5,5]$ when $e_{m}=1$.

$y_{l+1}^{2}=\alpha_{l} y_{l+1}+\beta_{l}$, which completes the creation of the $l$ th segment.

In LPLA, where a lower envelope needs to be produced, the first step for the derivation of segment $l$, starts from a point of maximum error, $\left\{y_{l}, y_{l}^{2}-e_{m}\right\}$ (initially, the procedure starts from $y_{1}=y_{L}-e_{m}$ ) and computes a line $\alpha_{l} y+b_{l}$ that is tangent to $y^{2}$. In the second step, a new point of maximum error is found such that $\alpha_{l} y_{l+1}+\beta_{l}=y_{l+1}^{2}-e_{m}$, which completes the creation of the $l$ th segment. UPLA and LPLA continue operation until $y_{U}$ is reached. Fig. 2 illustrates an example of the upper and lower envelope approximation functions produced for $f(x)=x^{2}$ using the described methods when $e_{m}=1$.

One issue that still has not been discussed concerns the region of approximation. Since the approximated functions $\left(y_{i}^{R}\right)^{2}$ and $\left(y_{i}^{I}\right)^{2}, i \in \mathcal{K}$ are produced from $\left\|\tilde{\mathbf{h}}_{i}^{T} \mathbf{w}\right\|^{2}=$ $\mathbf{w}^{H} \tilde{\mathbf{h}}_{i}^{*} \tilde{\mathbf{h}}_{i}^{T} \mathbf{w}$, an upper bound for both functions can be established by finding the maximum value of this term. Based on the fact that a feasible solution $\hat{\mathbf{w}}$ provides an upper bound solution to (14), $f_{U B}=\hat{\mathbf{w}}^{H} \hat{\mathbf{w}}$, e.g. obtained from (25), an upper bound for $\left(y_{i}^{R}\right)^{2}$ and $\left(y_{i}^{I}\right)^{2}$ is the quantity $f_{U B} \lambda_{\max }\left(\tilde{\mathbf{h}}_{i}^{*} \tilde{\mathbf{h}}_{i}^{T}\right)$ obtained from the maximum Rayleigh quotient. Since $y_{i}^{R} \geq 0$, the approximation region of $y_{i}^{R}$ is $\left[0, \sqrt{f_{U B} \lambda_{\max }\left(\tilde{\mathbf{h}}_{i}^{*} \tilde{\mathbf{h}}_{i}^{T}\right)}\right]$ and of $y_{i}^{I}$ is $\left[-\sqrt{f_{U B} \lambda_{\max }\left(\tilde{\mathbf{h}}_{i}^{*} \tilde{\mathbf{h}}_{i}^{T}\right)}, \sqrt{f_{U B} \lambda_{\max }\left(\tilde{\mathbf{h}}_{i}^{*} \tilde{\mathbf{h}}_{i}^{T}\right)}\right]$.

For the approximation of the nonconvex separable constraints with PLA functions we consider the multiple choice model [38]. Let us assume that function $\left(y_{i}^{R}\right)^{2}\left[\left(y_{i}^{I}\right)^{2}\right]$ is approximated by $L_{i}^{R} \quad\left[L_{i}^{I}\right]$ linear segments with slopes $\alpha_{i, 1}^{R}, \ldots, \alpha_{i, L_{i}^{R}}^{R}$, zero-crossing points $\beta_{i, 1}^{R}, \ldots, \beta_{i, L_{i}^{R}}^{R}$ and breakpoints ${ }^{1} \gamma_{i, 1}^{R}, \ldots, \gamma_{i, L_{i}^{R}+1}^{R}$. For the approximation of $\left(y_{i}^{R}\right)^{2}$, the multiple choice model introduces binary variables $\psi_{i, l}^{R}$ to indicate whether the $l$ th linear segment $\left[\gamma_{i, l}^{R}, \gamma_{i, l+1}^{R}\right]$ is active and continuous variables $\xi_{i, l}^{R}$, such that $\xi_{i, l}^{R}=y_{i}^{R}$ if the $l$ th segment is active and zero otherwise. As a result, it is true that $\left(y_{i}^{R}\right)^{2} \approx \sum_{l} \alpha_{i, l}^{R} \xi_{i, l}^{R}+\beta_{i, l}^{R} \psi_{i, l}^{R}$ and $y_{i}^{R}=\sum_{l} \xi_{i, l}^{R}$. The

\footnotetext{
${ }^{1}$ The corresponding quantities for $\left(y_{i}^{I}\right)^{2}$ have $I$ instead of $R$ in the exponent.
}

same analysis holds true for the approximation of $\left(y_{i}^{I}\right)^{2}$.

Based on the above, the resulting MISOCP formulation is:

$$
\begin{aligned}
& \min _{\left\{\mathbf{w}, \boldsymbol{\rho}, \boldsymbol{\rho}_{2}, \mathbf{z}_{i}^{ \pm}, \mathbf{r}_{1}, \mathbf{r}_{2}, \mathbf{u}, \mathbf{y}^{R}, \mathbf{y}^{I}, \mathbf{v}, \Psi^{R}, \Psi^{I}, \mathbf{y}^{R}, \mathbf{y}^{I}\right\}}\|\mathbf{w}\|^{2} \\
& \text { s.t. Constraints (15)-(19), (21)-(23), (14d) } \\
& u_{i}=\sum_{l=1}^{L_{i}^{R}}\left(\alpha_{i, l}^{R} \xi_{i, l}^{R}+\beta_{i, l}^{R} \psi_{i, l}^{R}\right) \\
& +\sum_{l=1}^{L_{i}^{I}}\left(\alpha_{i, l}^{I} \xi_{i, l}^{I}+\beta_{i, l}^{I} \psi_{i, l}^{I}\right), i \in \mathcal{K} \\
& y_{i}^{R}=\sum_{l=1}^{L_{i}^{R}} \xi_{i, l}^{R}, y_{i}^{I}=\sum_{l=1}^{L_{i}^{I}} \xi_{i, l}^{I}, i \in \mathcal{K} \\
& \psi_{i, l}^{R} \beta_{i, l}^{R} \leq \xi_{i, l}^{R} \leq \psi_{i, l+1}^{R} \beta_{i, l+1}^{R}, l=1, \ldots, L_{i}^{R}, i \in \mathcal{K} \\
& \psi_{i, l}^{I} \beta_{i, l}^{I} \leq \xi_{i, l}^{I} \leq \psi_{i, l+1}^{I} \beta_{i, l+1}^{I}, l=1, \ldots, L_{i}^{I}, i \in \mathcal{K} \\
& \rho_{2 i}=1-\rho_{i}, i \in \mathcal{K} \\
& u_{i} \rho_{2 i} \geq E_{i}, i \in \mathcal{K} \\
& \psi_{i, l}^{R} \in\{0,1\}, l=1, \ldots, L_{i}^{R}, i \in \mathcal{K} \\
& \psi_{i, l}^{I} \in\{0,1\}, l=1, \ldots, L_{i}^{I}, i \in \mathcal{K} .
\end{aligned}
$$

\section{THEORETICAL ANALYSIS}

In this section, the feasibility of problem (14) and the asymptotic optimality of the developed algorithms is investigated.

\section{A. Feasibility Analysis}

The following theorem establishes the main result on the feasibility test, which reduces to the solution of an LP constraint satisfaction problem.

Theorem 1. Let $\Delta_{i}=\Gamma_{i}\left(N_{0}+N_{C}\right)$. Problem (14) is feasible if and only if LP problem (40) is feasible.

find $\{\mathbf{w}\}$

$$
\begin{array}{r}
\text { s.t. } \operatorname{Re}\left(\tilde{\mathbf{h}}_{i}^{T} \mathbf{w}\right)-\operatorname{Im}\left(\tilde{\mathbf{h}}_{i}^{T} \mathbf{w}\right) / \tan \theta \geq \sqrt{\Delta_{i}}, i \in \mathcal{K} \\
\quad \operatorname{Re}\left(\tilde{\mathbf{h}}_{i}^{T} \mathbf{w}\right)+\operatorname{Im}\left(\tilde{\mathbf{h}}_{i}^{T} \mathbf{w}\right) / \tan \theta \geq \sqrt{\Delta_{i}}, i \in \mathcal{K}
\end{array}
$$

Proof: The proof is provided in Appendix A.

Theorem 1 states that although the problem is nonlinear and non-convex its feasibility can be tested through the solution of an LP problem. In fact, Theorem 40 confirms that the feasibility of problem (14) is independent of the EH constraints and of the $\rho_{i}$ parameters, similar to other works on MISO broadcast channels [4]. Theorem 1 can be exploited to test if an examined problem instance is feasible in a fast manner, as the solution of the resulting LP formulation is significantly faster compared to the developed algorithms in Sections IV VI which require the solution of SOCP or SDP problems with a larger number of variables.

\section{B. Asymptotic optimality}

The developed algorithms are suboptimal in general. However, we prove that they asymptotically converge to the optimal solution when the SINR threshold $\left(\Gamma_{i}\right)$ and the modulation 
order $(M)$ tends to infinity. Towards this direction note that Algorithm 1 always provides equal or better bounds than the SOCP algorithm based on (25), while the solution from (38) provides a tighter LB than (35). Hence, it suffices to establish the result for (25) and (35), in order for it to hold true for Alg. 1 and formulation (38). The main result is as follows:

Proposition 1. The solutions of (25) and (35) asymptotically converge to the optimum for $M \rightarrow \infty$ and for $\Gamma_{i} \rightarrow \infty, i \in \mathcal{K}$.

Proof: The proof is provided in Appendix B.

Proposition 1 is important because practical systems often use a large modulation order and high SINR threshold to achieve high data rates and low outage probability, in which cases the proposed algorithms will achieve asymptotically optimal results. The simulation section verifies this result for a practical communication scenario.

\section{Simulation RESUlts}

In this section we investigate the benefits of using CI, examine the performance of the developed algorithms and experimentally verify the theoretical findings.

The considered setting involves $K$ receivers randomly located around the $\mathrm{BS}$ with distance $l_{i}$ and direction $\zeta_{i}$ drawn from the uniform distribution, $l_{i} \sim U(2,7) \mathrm{m}$ and $\zeta_{i} \sim U(-\pi, \pi)$. Each receiver can harvest energy at frequency $f=915 \mathrm{MHz}$ while it is assumed that the gains per antenna at the $\mathrm{BS}$ and receivers are $8 \mathrm{dBi}$ and $3 \mathrm{dBi}$, respectively. The path attenuation of receiver $i, L_{i}$, is obtained using the Friis equation with reference distance $1 \mathrm{~m}$ and path loss coefficient 2.5. It is further assumed that $K=N=4, N_{0}=-70 \mathrm{dBm}$ and $N_{C}=-50 \mathrm{dBm}$, while the EH and SINR thresholds are the same for all receivers, i.e. $\Gamma_{i}=\Gamma, E_{i}=E, i \in \mathcal{K}$. The modulation scheme used is QPSK unless otherwise stated. Rician fading is used to model the channel as the short distance between the BS and the receivers implies dominance of the line-of-sight signal. Hence, $\mathbf{h}_{i}$ is composed of the line-ofsight (LOS) signal, $\mathbf{h}_{i}^{L O S}$ and the non-LOS signal $\mathbf{h}_{i}^{N L O S}$ according to the expression [4]

$$
\mathbf{h}_{i}=\sqrt{\frac{K_{R}}{1+K_{R}}} \mathbf{h}_{i}^{L O S}+\sqrt{\frac{1}{1+K_{R}}} \mathbf{h}_{i}^{N L O S},
$$

where $K_{R}=5 \mathrm{~dB}$ is the Rician factor. For the LOS signal the far-field uniform linear antenna array model with $\lambda / 2$ distance between antenna elements is considered [39] which implies that $\mathbf{h}_{i}^{L O S}=\sqrt{L_{i}}\left[1, \mathrm{e}^{-j\left(1 \pi \sin \zeta_{i}\right)}, \ldots, \mathrm{e}^{-j\left((N-1) \pi \sin \zeta_{i}\right)}\right]^{T}$. Rayleigh fading is adopted for the NLOS signal, $\mathbf{h}_{i}^{N L O S} \in$ $\mathbb{C}^{N \times 1}$ which means that each of its elements are circularly symmetric complex Gaussian (CSCG) random variables with zero mean and variance $L_{i}$.

The performance of nine algorithms is examined:

1) SDPnoCI: The conventional SDP algorithm based on (8).

2) SOCP-UB: The SOCP algorithm based on (25).

3) ISOCP-UB: SOCP-UB followed by the bisection method of Section IV-C.

4) SLA-UB: The iterative SLA Algorithm 1.

5) MISOCP-UB: Formulation (39) along with LPLA.

6) MISOCP-LB: Formulation (39) along with UPLA.

7) SDP-LB: The SDP algorithm based on (37).

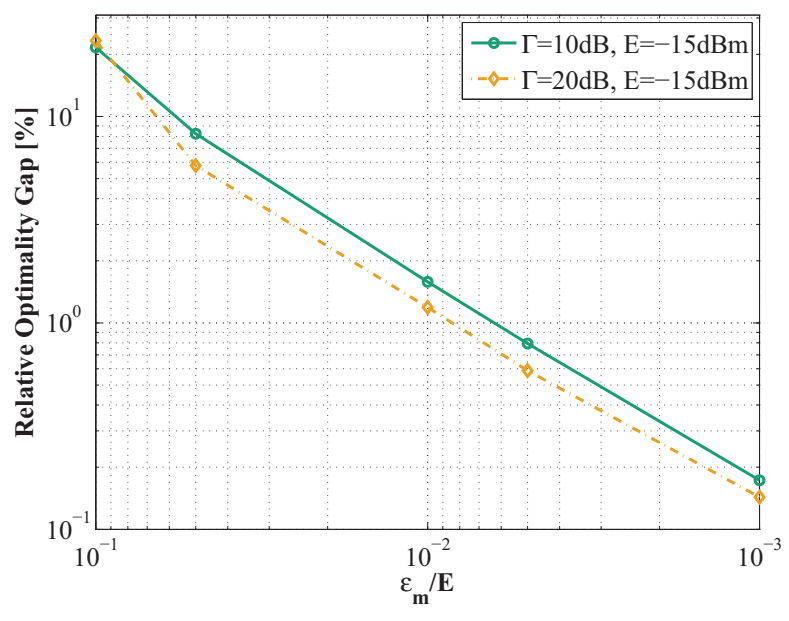

Fig. 3. Relative percentage gap between the solution of MISOCP-UB and MISOCP-LB for $E=-15 \mathrm{dBm}$ and $\Gamma=\{10,20\} \mathrm{dB}$ when $K=4$.

8) ISDP-LB: The SDP algorithm based on (38).

9) SOCP-LB: The SOCP algorithm based on (35).

Note that the first five algorithms provide upper bounds and the last four lower bounds to the optimal solution. Furthermore, all figures depict results that are averaged over 1000 randomly generated problem instances per examined case.

First, we investigate the tightness of the exponential complexity algorithm MISOCP according to (39). As noted above, if LPLA is used then the expression $\left(y_{k}^{R}\right)^{2}+\left(y_{k}^{I}\right)^{2}$ will be larger than any piecewise linear approximating function, so that the solution of (39) will provide an UB to the original solution; similarly, applying UPLA provides a LB to the optimal solution. Fig. 3 depicts the relative percentage optimality gap between the MISOCP-UB and MISOCP-LB as a function of $e_{m} / E$. The gap is defined as

$$
\frac{\left(\left\|\mathbf{w}^{\mathrm{UB}}\right\|_{2}^{2}-\left\|\mathbf{w}^{\mathrm{LB}}\right\|_{2}^{2}\right)}{\left\|\mathbf{w}^{\mathrm{LB}}\right\|_{2}^{2}} \times 100
$$

where $\mathbf{w}^{\mathrm{UB}}$ and $\mathrm{w}^{\mathrm{LB}}$ denote the solution obtained from MISOCP-UB and MISOCP-LB, respectively. From the figure it is clear that the gap closes for decreasing value of $e_{m}$, so that for $e_{m}=0.001 E$ the gap is about $0.1 \%$. Nonetheless, as smaller $e_{m}$ values result in more piecewise linear segments, for the rest of the experiments we have selected $e_{m}=0.01 E$ which yields an optimality gap less than $2 \%$.

Figs. 4, 5 and 6 depict the total transmitted power achieved by the different investigated algorithms with varying $\Gamma, E$ and $K$, respectively. In these figures, continuous/dashed lines represent the performance of upper/lower bounding algorithms, while the dotted line represents the optimal solution of the conventional scheme with no CI (i.e., SDPnoCI). From the figures it can be seen that ISOCP-UB performs better than SOCP-UB by up to $0.8 \mathrm{dBW}$, while the best performing UB algorithm is SLA-UB which achieves performance at most $1.5 \mathrm{dBW}$ better than ISOCP-UB. Regarding the LB algorithms, clearly SDP provides a very bad LB, contrary to conventional precoding where it provides optimal results, while ISDP and SOCP-LB have almost identical performance, having a 

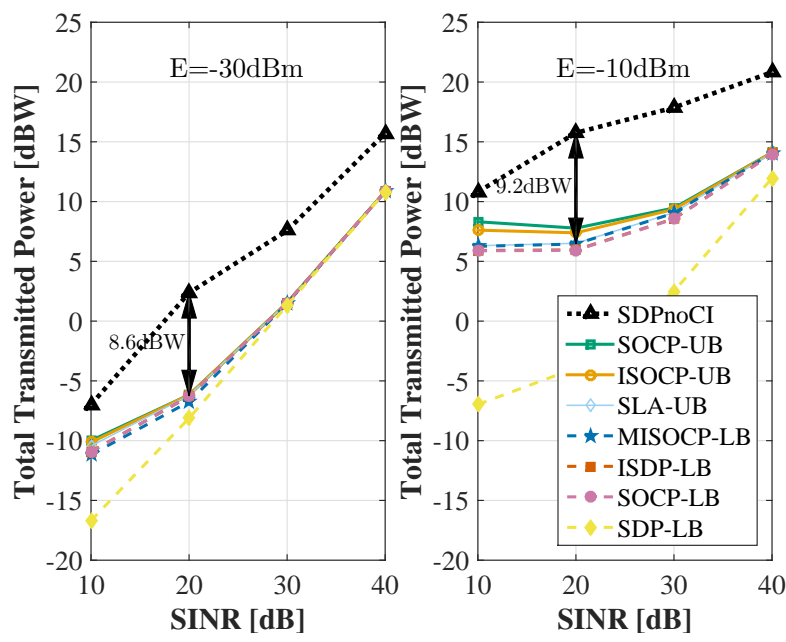

Fig. 4. Total transmitted power of investigated algorithms for varying $\Gamma$ when $E=\{-30,-10\} \mathrm{dBm}$ and $K=4$.

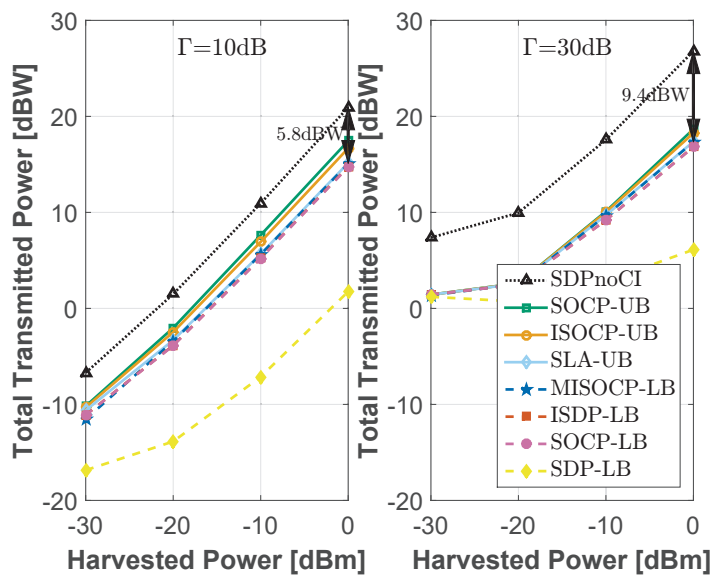

Fig. 5. Total transmitted power of investigated algorithms for varying $E$ when $\Gamma=\{10,30\} \mathrm{dB}$ and $K=4$.

$0.5 \mathrm{dBW}$ gap compared to the tight MISOCP-LB in the worst case. Another important observation is that the performance of the conventional precoding scheme SDPnoCI is significantly worse than SLA-UB which is the best CI precoding algorithm. In fact, for varying $\Gamma, E$ and $K$ there is a performance gap between SLA-UB and SDPnoCI in the range [3.5, 9.2] dBW, $[3.9,9.4] \mathrm{dBW}$ and $[3.8,13.3] \mathrm{dBW}$, respectively. In addition, Fig. 4 indicates that while SINR increases, the gap between the upper and lower bounding algorithms tends to zero, as expected from Proposition 1. Furthermore, Fig. 6 indicates that the relative performance of different algorithms remains the same for increasing number of users.

Figs. 7 and 8 depict the relative percentage optimality gap between SLA-UB, which is the best performing polynomial complexity algorithm, and the best lower bound for varying
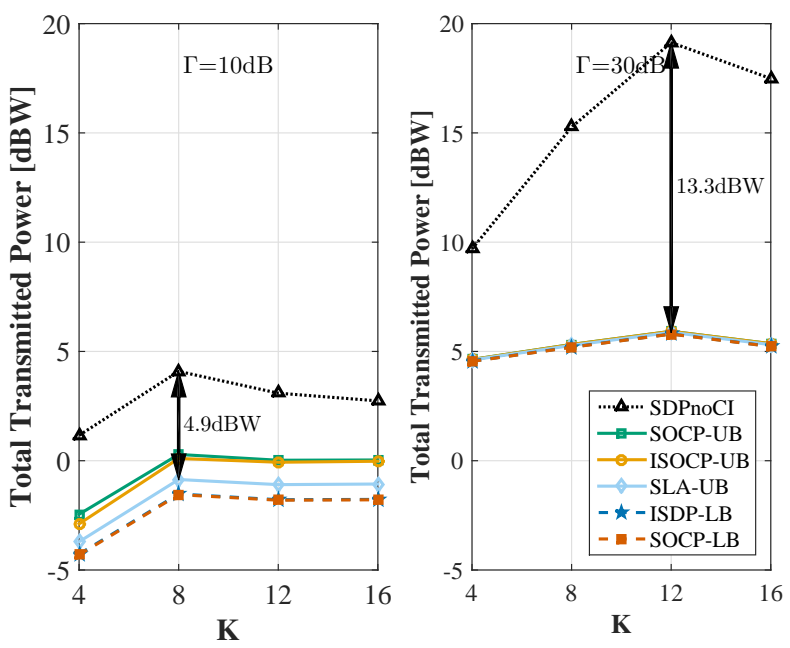

Fig. 6. Total transmitted power of investigated algorithms for varying $K$ when $\Gamma=\{10,30\} \mathrm{dB}$ and $E=-20 \mathrm{dBm}$; here, we consider $N=K$.

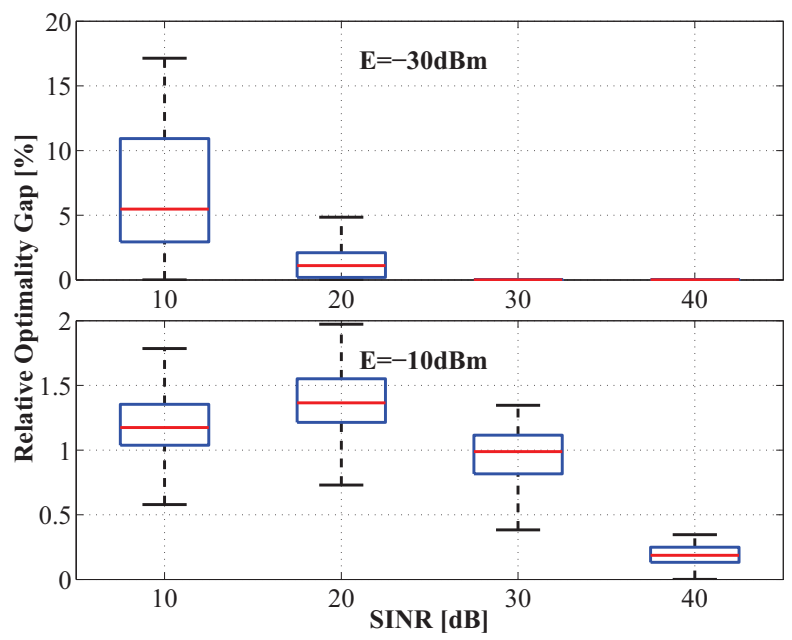

Fig. 7. Relative percentage optimality gap between SLA-UB and the best lower bound for varying $\operatorname{SINR}(\Gamma)$ when $E=\{-30,-10\} \mathrm{dBm}$ and $K=4$.

$\Gamma$ and $E$ in the form of boxplots ${ }^{2}$. As can be seen, all cases examined yield median performance within 5\% from the optimal, while in the majority of the cases the optimality gap is less than $2 \%$. Also apart from the case $\Gamma=10 \mathrm{~dB}$ and $E=-30 \mathrm{dBm}$ all other cases have small dispersion from the median value which is less than $5 \%$ for $E=-30 \mathrm{dBm}$ and less than $2.5 \%$ in all other cases examined. Note that performance is observed for $e_{m}=0.01 E$, which yielded a relative gap between MISOCP-UB and MISOCP-LB approximately $2 \%$ which implies that SLA-UB is even closer to optimality. CI approaches are superior to the conventional precoding approach, SDPnoCI, not only in terms of total transmitted power, but also in terms of computational complexity as it results in SOCP instead of SDP formulations. This is shown

\footnotetext{
${ }^{2}$ The bottom and top of each box indicate the first and third quartiles $(25 \%$ and $75 \%)$ of a ranked data set, while the horizontal line inside the box indicates the median value (second quartile). The horizontal lines outside the box indicate the lowest/highest datum still within 1.5 inter-quartile range of the lower/upper quartile; for normally distributed data this corresponds to approximately $0.35 \% / 99.65 \%$.
} 


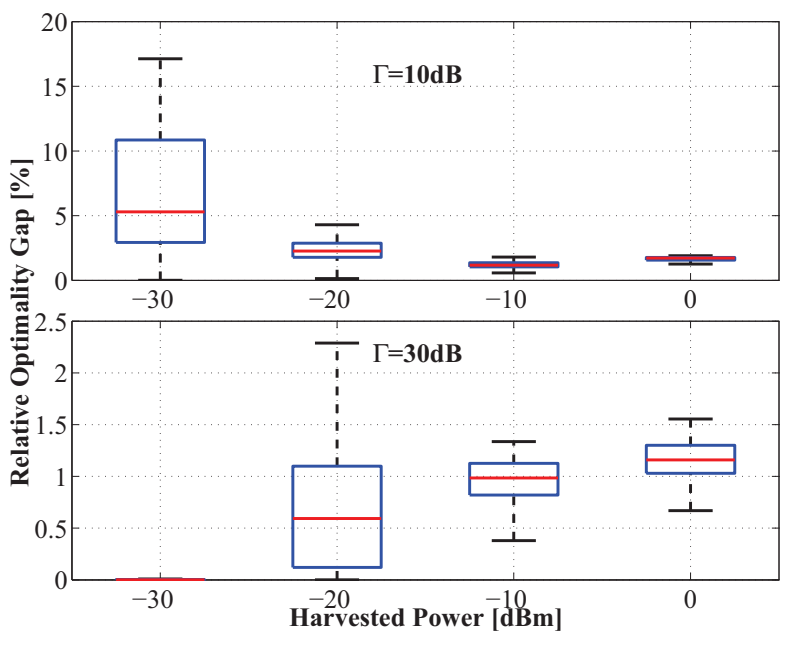

Fig. 8. Relative percentage optimality gap between SLA-UB and the best lower bound for varying $E$ when $\Gamma=\{10,30\} \mathrm{dB}$ and $K=4$.
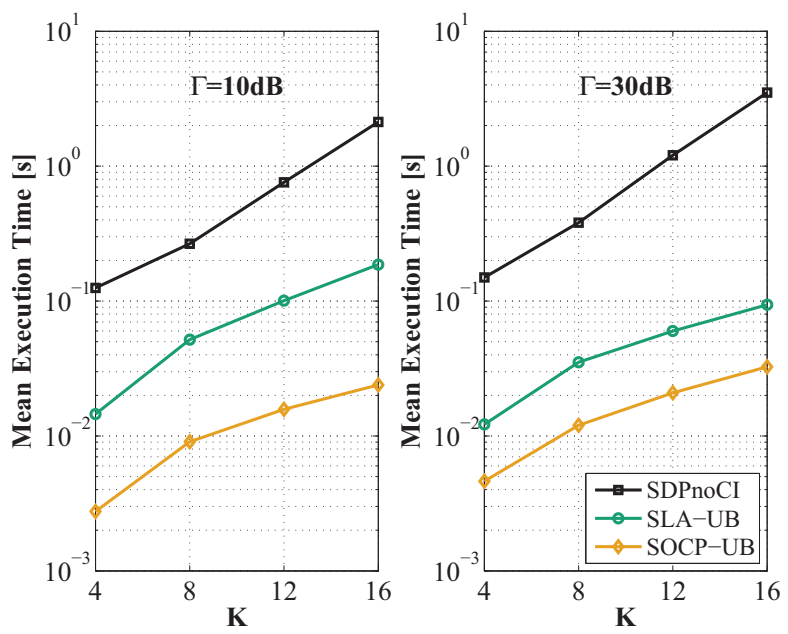

Fig. 9. Execution time of SDPnoCI, SOCP-UB and SLA-UB for varying $K$ when $E=-20 \mathrm{dBm}$ and $\Gamma=\{10,30\} \mathrm{dB}$.

in Fig. 9 that depicts the execution time of SDPnoCI, SLAUB and SOCP-UB. From the figure it is clear that SLA-UB is more than one order of magnitude faster than SDPnoCI, but also slower than SOCP-UB by 3-5 times. This implies that between the two CI-based approaches there is a tradeoff between computational complexity and optimality as SOCPUB is faster but has inferior performance compared to SLAUB.

We have already illustrated in Figs. 4 and 7 that as the SINR threshold increases the performance gap between UB and LB algorithms tends to zero. To demonstrate the fact that the same is true for the modulation order $M$, we depict in Fig. 10 the relative percentage gap between the solution of SOCPUB and SOCP-LB as the modulation order increases from $M=2$ (BPSK) to $M=32$ (32-PSK). It can be observed that the optimality gap reduces by four orders of magnitude as we increase $M$, reaching an optimality gap of less than $1 \%$ for all examined cases.

Finally, Fig. 11 compares the relative percentage perfor-

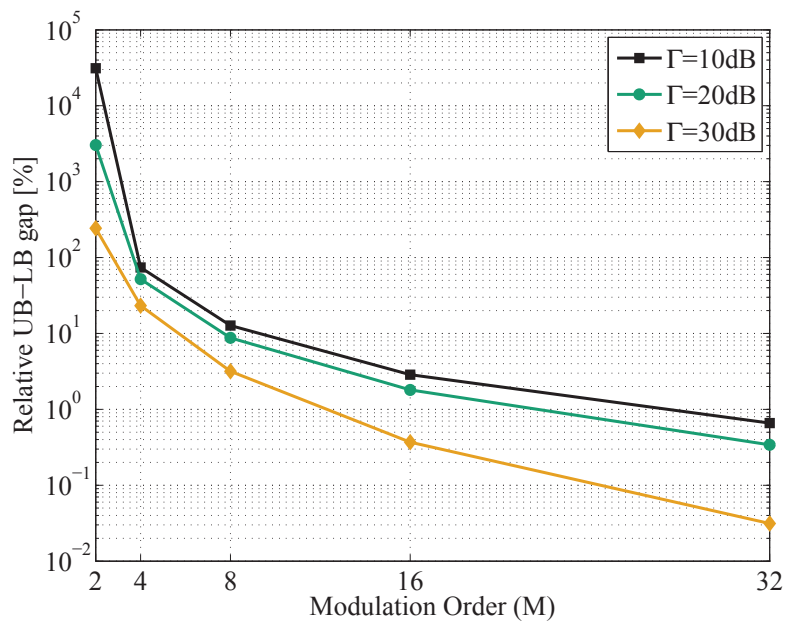

Fig. 10. Relative percentage gap between the solution of SOCP-UB and SOCP-LB varying $\Gamma$ when $E=-10 \mathrm{dBm}$ and $K=8$.

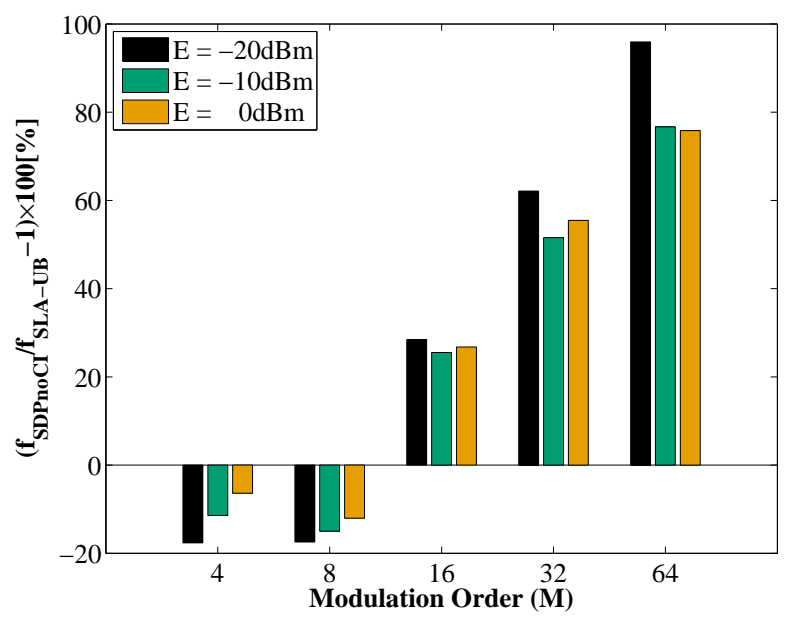

Fig. 11. Relative percentage performance comparison between SDPnoCI and SLA-UB in terms of transmit power for varying modulation order, $M$ and $\Gamma=M-1$ for $E=\{-20,-10,0\} \mathrm{dBm}$ and $K=N=8$; negative (positive) values imply that SDPnoCI (SLA-UB) is better, i.e. requires less transmit power.

mance between SDPnoCI and SLA-UB in terms of total transmit power for varying modulation order, $M$, and $\Gamma=M-1$, so that both algorithms have $\log _{2}(M)$ bit $/ \mathrm{s} / \mathrm{Hz}$ theoretical maximum spectral efficiency. It is clear that SDPnoCI provides up to $20 \%$ better results for $M=4$ and $M=8$, while for higher modulation order, and hence higher SINR, the SLAUB algorithm is significantly better requiring up to half total transmit power compared to SDPnoCI.

\section{CONCLUSiOnS}

In this paper, we have explored for the first time, the exploitation of the constructive interference in MISO downlink to boost the performance for both information decoding and energy harvesting. We have shown that, by means of data-aided beamforming, constructive interference can be exploited to improve the signal power as well as act as a source of wireless power transfer. Despite the fact that the formulated problem is nonconvex, several polynomial complexity algorithms have 
been developed that provide results close to optimality, as well as reduced transmition power by $4-13 \mathrm{dBW}$ and one to two orders of magnitude faster execution compared to conventional precoding design. In addition, it has been theoretically proven that the proposed algorithms asymptotically converge to the optimal solution when the SINR threshold and the modulation order tend to infinity.

\section{APPENDIX A PROOF OF THEOREM 1}

In order to prove this theorem we need to first prove that problem (14) is feasible if and only if (43) is feasible.

$$
\begin{aligned}
& \text { find } \mathbf{w} \\
& \text { s.t. } \operatorname{Re}\left(\tilde{\mathbf{h}}_{i}^{T} \mathbf{w}\right)-\left|\operatorname{Im}\left(\tilde{\mathbf{h}}_{i}^{T} \mathbf{w}\right)\right| / \tan \theta \geq \sqrt{\Delta_{i}}, i \in \mathcal{K}
\end{aligned}
$$

This result is proven following a similar procedure to the one taken for the proof of Lemmas 3.1 and 3.2 in [4] and for this reason is omitted due to space limitations. Hence, to prove this theorem, we simply need to eliminate the absolute value operator from (43b). Towards this direction note that $\left|\operatorname{Im}\left(\tilde{\mathbf{h}}_{i}^{T} \mathbf{w}\right)\right|=\max \left\{\operatorname{Im}\left(\tilde{\mathbf{h}}_{i}^{T} \mathbf{w}\right),-\operatorname{Im}\left(\tilde{\mathbf{h}}_{i}^{T} \mathbf{w}\right)\right\}$ and that constraints (40b)-(40c) ensure that:

$$
\operatorname{Re}\left(\tilde{\mathbf{h}}_{i}^{T} \mathbf{w}\right) \geq \max \left\{\operatorname{Im}\left(\tilde{\mathbf{h}}_{i}^{T} \mathbf{w}\right),-\operatorname{Im}\left(\tilde{\mathbf{h}}_{i}^{T} \mathbf{w}\right)\right\}+\sqrt{\Delta_{i}},
$$

which is equivalent to (43b). This completes the proof.

\section{APPENDIX B \\ PROOF OF PROPOSITION 1}

First we prove asymptotic optimality for the modulation order. Since the solution of (25) provides an UB and the solution of (35) a LB, it suffices to show that as $M \rightarrow \infty$, the two formulations become identical which means that the gap between a feasible solution and a lower bound solution is equal to zero. Observing the approximated $\mathrm{EH}$ terms from formulations (25) and (35) is can be easily deduced that

$$
\left(y_{i}^{R}\right)^{2} \leq\left(y_{i}^{R}\right)^{2}+\left(y_{i}^{I}\right)^{2} \leq y_{i}^{R}\left(y_{i}^{R}+\left|y_{i}^{I}\right| \tan \theta\right)
$$

For $M \rightarrow \infty$ it is true that $\tan \theta \rightarrow 0$ which means that $\left(y_{i}^{R}\right)\left(y_{i}^{R}+\left|y_{i}^{I}\right| \tan \theta\right) \rightarrow\left(y_{i}^{R}\right)^{2}$, and based on (45) this also implies that $\left|y_{i}^{I}\right| \rightarrow 0$. This is also verified from the SINR constraint as for $\tan \theta \rightarrow 0$ it must be true that $\left|y_{i}^{I}\right| \leq \mu, \mu \rightarrow 0$, which holds true when $\left|y_{i}^{I}\right| \rightarrow 0$. Hence, we have proven that for $M \rightarrow \infty$ the UB and LB EH constraints tend to the accurate EH constraint, completing the proof for the modulation order.

Regarding asymptotic optimality for $\Gamma_{i} \rightarrow \infty, i \in \mathcal{K}$, it is true from the feasibility analysis of the SINR constraints (see (43b)) that $y_{i}^{R} \geq\left|y_{i}^{I}\right| / \tan \theta+\sqrt{\Delta_{i}}$, where $\Delta_{i} \rightarrow \infty$. Hence, the expression $y_{i}^{R}+\left|y_{i}^{I}\right| \tan \theta \rightarrow y_{i}^{R}$ so that $y_{i}^{R}\left(y_{i}^{R}+\right.$ $\left.\left|y_{i}^{I}\right| \tan \theta\right) \rightarrow\left(y_{i}^{R}\right)^{2}$. This shows that for $\Gamma_{i} \rightarrow \infty$ the upper and lower bound formulations ((25) and (35)) will provide the same solution which implies optimality, completing the proof.

\section{REFERENCES}

[1] G. Zheng, C. Masouros, I. Krikidis and S. Timotheou, "Exploring Green Interference Power for Wireless Information and Energy Transfer in the MISO Downlink," in IEEE International Conference on Communications (ICC), London, UK, June 2015.

[2] P. Grover and A. Sahai, "Shannon meets Tesla: wireless information and power transfer," in Proc. IEEE Int. Symp. Inf. Theory, Austin, USA, June 2010, pp. 2363-2367.

[3] R. Zhang and C. K. Ho, "MIMO broadcasting for simultaneous wireless information and power transfer," IEEE Trans. Wireless Commun., vol. 12, no. 5, pp. 1989-2001, May 2013.

[4] Q. Shi, L. Liu, W. Xu, and R. Zhang, "Joint transmit beamforming and receive power splitting for MISO SWIPT systems," IEEE Trans. Wireless Commun., vol, 13, no. 6, pp. 3269-3280, June 2014.

[5] J. Park and B. Clerckx, "Joint wireless information and energy transfer in a two-user MIMO interference channel," IEEE Trans. Wireless Commun., vol. 12, no. 8, pp. 4210-4221, Aug. 2013.

[6] S. Timotheou, I. Krikidis, G. Zheng, and B. Ottersten, "Beamforming for MISO interference channels with QoS and RF energy transfer," IEEE Trans. Wireless Commun., vol. 13, no. 5, pp. 2646-2658, May 2014.

[7] Q. Shi, W. Xu, T-H Chang, Y. Wang and E. Song, "Joint Beamforming and Power Splitting for MISO Interference Channel With SWIPT: An SOCP Relaxation and Decentralized Algorithm," IEEE Trans. Signal Processing, vol.62, pp. 6149-6208, 2014.

[8] D.W.K. Ng, E. S. Lo, and R. Schober, "Wireless Information and Power Transfer: Energy Efficiency Optimization in OFDMA Systems," IEEE Trans. on Wireless Commun., vol. 12, no. 12, pp. 6352-6370, Dec. 2013.

[9] S. He, Y. Huang, W. Chen, S. Jin, H. Wang, and L. Yang, "Energy Efficient Coordinated Precoding Design for Multi- cell System with RF Energy Harvesting," EURASIP Journal on Wireless Communications and Networking, March 2015, 2015:67.

[10] M. Bengtsson and B. Ottersten, "Optimal and suboptimal transmit beamforming," in Handbook of Antennas in Wireless Communications, L. Godara, Ed. Boca Raton, FL: CRC Press, ch. 18, 2001.

[11] A. Wiesel, Y. C. Eldar, and S. Shamai, "Optimization of the MIMO compound capacity," IEEE Trans. Wireless Commun., vol. 6, no. 3, pp. 1094-1101, Mar. 2007

[12] Q. Shi, M. Razaviyayn, Z. Q. Luo, and C. He, "An Iteratively Weighted MMSE Approach to Distributed Sum-Utility Maximization for a MIMO Interfering Broadcast Channel," IEEE Trans. Signal Process., vol.59, no.9, pp.4331,4340, Sept. 2011

[13] W. Yu, T. Kwon, and C. Shin, "Multicell Coordination via Joint Scheduling, Beamforming, and Power Spectrum Adaptation," IEEE Trans. Wireless Commun., vol.12, no.7, pp.1,14, July 2013

[14] D. Nguyen, L. Tran, P. Pirinen, and M. Latva-aho, "Precoding for Full Duplex Multiuser MIMO Systems: Spectral and Energy Efficiency Maximization," IEEE Trans. Signal Process., vol. 61, no. 16, pp. 4038 - 4050, Aug. 2013

[15] A. Pascual-Iserte, D. P. Palomar, A. I. Perez-Neira, and M. A. Lagunas, "A robust maximin approach for MIMO communications with partial channel state information based on convex optimization", IEEE Trans. Signal Process., vol. 54, no. 1, pp. 346-360, Jan. 2006.

[16] J. Wolfowitz, Coding Theorems of Information Theory, 3rd ed. Berlin, Germany: Springer-Verlag, 1978.

[17] M. Biguesh, S. Shahbazpanahi, and A. B. Gershman, "Robust downlink power control in wireless cellular systems," EURASIP J. Wireless Commun. Netw., vol. 2004, no. 2, pp. 261-272, Dec. 2004.

[18] M. Payaro, A. Pascual, and M. Lagunas, "Robust power allocation designs for multiuser and multiantenna downlink communication systems through convex optimization," IEEE J. Sel. Areas Commun., vol. 25, no. 7, pp. 1390-1401, Sep. 2007.

[19] N. Vucic and H. Boche, "Robust QoS-constrained optimization of downlink multiuser MISO systems," IEEE Trans. Signal Proc., vol. 57, no. 2, pp. 714-725, Feb. 2009.

[20] G. Zheng, K. K. Wong and T. S. Ng, "Robust Linear MIMO in the Downlink: A Worst-Case Optimization with Ellipsoidal Uncertainty Regions," EURASIP Journal on Advances in Signal Processing, vol. 2008

[21] M. Schubert and H. Boche, "Solution of the Multi-User Downlink Beamforming Problem with Individual SINR Constraints," IEEE Trans. Veh. Tech., vol. 53, no. 1, pp. 18-28, Jan. 2004

[22] C. Masouros, and E. Alsusa, "Dynamic linear precoding for the exploitation of known interference in MIMO broadcast systems," IEEE Trans. Wireless Commun., vol. 8, no. 3, pp. 1396-1404, Mar. 2009. 
[23] C. Masouros, "Correlation rotation linear precoding for MIMO broadcast communications", IEEE Trans. Signal Process., vol. 59, no. 1, pp. 252 262, Jan. 2011.

[24] C. Masouros, T. Ratnarajah, M. Sellathurai, C. Papadias, and A. Shukla, "Known interference in wireless communications: a limiting factor or a potential source of green signal power?," IEEE Commun. Mag., vol. 51, no. 10, pp. 162-171, Oct. 2013.

[25] G. Zheng, I. Krikidis, C. Masouros, S. Timotheou, D. A. Toumpakaris, and Z. Ding, "Rethinking the Role of Interference in Wireless Networks," IEEE Commun. Mag., vol.52, no.11, pp.152,158, Nov. 2014

[26] M. Alodeh, S. Chatzinotas and B. Ottersten, "Constructive Multiuser Interference in Symbol Level Precoding for the MISO Downlink Channel," IEEE Trans. Signal Process., vol. 63 , no. 9 , pp. 2239-2252, May 2015

[27] C. Masouros and G. Zheng, "Exploiting Known Interference as Green Signal Power for Downlink Beamforming Optimization," IEEE Trans. Signal Process., vol.63, no.14, pp.3668-3680, July, 2015

[28] C. Masouros and E. Alsusa, "Soft Linear Precoding for the Downlink of DS/CDMA Communication Systems," IEEE Trans. on Veh. Tech., vol. 59, no. 1, pp. 203-215, Jan 2010

[29] M. Alodeh, S. Chatzinotas, and B. Ottersten, "Constructive Interference through Symbol Level Precoding for Multi-level Modulation," submitted to Globecom 2015, available on Arxiv in 28th April 2015.

[30] 3GPP TS 36.211, V8.2.0 (2008-03), Release 8 "Evolved Universal Terrestrial Radio Access (E-UTRA); Physical Channels and Modulation"

[31] F. Rusek, D. Persson, B. K. Lau, E. Larsson, T. Marzetta, O. Edfors, and F. Tufvesson, "Scaling Up MIMO: Opportunities and Challenges with Very Large Arrays," IEEE Signal Process. Magazine, vol.30, no.1, pp.40-60, Jan. 2013

[32] N. D. Sidiropoulos, T. N. Davidson, and Z. Q. Luo, "Transmit beamforming for physical-layer multicasting," IEEE Trans. Signal Proc., vol. 54, no. 6, pp. 2239-2251, June 2006.

[33] F. Alizadeh and D. Goldfarb, "Second-order cone programming," Mathematical Programming, Series B, 95:3-51, 2001.

[34] A. Ben-Tal and A. Nemirovski, Lectures on modern convex optimization: analysis, algorithms and engineering applications. MPS-SIAM Series on Optimization, SIAM, Philadelphia, 2001.

[35] L. Tran, M. Hanif, and M. Juntti, "A conic quadratic programming approach to physical layer multicasting for large-scale antenna arrays," IEEE Signal. Process. Lett., vol. 21, no. 1, pp. 114-117, Jan. 2014.

[36] S. Boyd and L. Vandenberghe, "Convex Optimization," Cambridge University Press, 2004.

[37] S. Timotheou, "Asset-task assignment algorithms in the presence of execution uncertainty," The Computer Journal, vol. 54, no. 9, pp. 15141525,2011

[38] J. P. Vielma, S. Ahmed, and G. Nemhauser, "Mixed-integer models for nonseparable piecewise-linear optimization: Unifying framework and extensions," Operations research, vol. 58, no. 2, pp. 303-315, 2010.

[39] E. Karipidis, N. D. Sidiropoulos, and Z. Q. Luo, "Far-field multicast beamforming for uniform linear antenna arrays," IEEE Trans. Signal Process., vol. 55, no. 10, pp. 4916-4927, 2007.

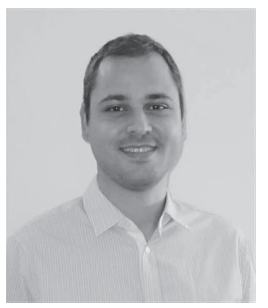

Stelios Timotheou (S'04-M'10) received a B.Sc. from the Electrical and Computer Engineering (ECE) School of the National Technical University of Athens, and an M.Sc. and Ph.D. from the Electrical and Electronic Engineering Department of Imperial College London. He is currently a Research Associate at the KIOS Research Center for Intelligent Systems and Networks of the University of Cyprus (UCY). In previous appointments, he was a Visiting Lecturer at the ECE Department of UCY, a Research Associate at the Computer Laboratory of the University of Cambridge and a Visiting Scholar at the Intelligent Transportation Systems Center \& Testbed, University of Toronto. His research focuses on the modeling and system-wide solution of problems in complex and uncertain environments that require real-time and close to optimal decisions by developing optimisation, machine learning and computational intelligence techniques. Application areas of his work include communication systems, intelligent transportation systems, disaster management and neural networks. $\mathrm{He}$ is a member of the IEEE and the ACM.

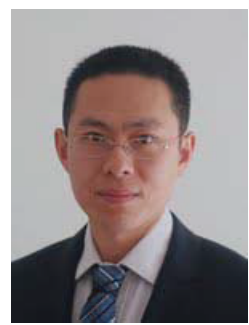

Gan Zheng (S'05-M'09-SM'12) received the BEng and the MEng from Tianjin University, Tianjin, China, in 2002 and 2004, respectively, both in Electronic and Information Engineering, and the $\mathrm{PhD}$ degree in Electrical and Electronic Engineering from The University of Hong Kong in 2008. He is currently a Senior Lecturer in the Wolfson School of Mechanical, Electrical and Manufacturing Engineering, Loughborough University, UK. His research interests include MIMO precoding, cooperative communications, cognitive radio, physical-layer security, full-duplex radio and energy harvesting. Dr. Zheng is a Senior Member of IEEE. He is the first recipient for the 2013 IEEE Signal Processing Letters Best Paper Award, and he also received 2015 GLOBECOM Best Paper Award.

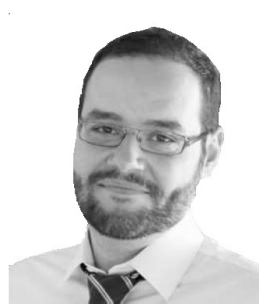

Christos Masouros (M'06, SM'14), is currently a Lecturer in the Dept. of Electrical \& Electronic Eng., University College London.

He received his Diploma in Electrical \& Computer Engineering from the University of Patras, Greece, in 2004, MSc by research and PhD in Electrical \& Electronic Engineering from the University of Manchester, UK in 2006 and 2009 respectively. He has previously held a Research Associate position in University of Manchester, UK and a Research Fellow position in Queen's University Belfast, UK.

He holds a Royal Academy of Engineering Research Fellowship 20112016 and is the principal investigator of the EPSRC project EP/M014150/1 on large scale antenna systems. His research interests lie in the field of wireless communications and signal processing with particular focus on Green Communications, Large Scale Antenna Systems, Cognitive Radio, interference mitigation techniques for MIMO and multicarrier communications. He was awarded the Best Paper Award in IEEE GlobeCom 2015 for his work on interference exploitation. He is an Associate Editor for IEEE Communications Letters, and was named Exemplary Reviewer for IEEE Transactions on Communications in 2015.

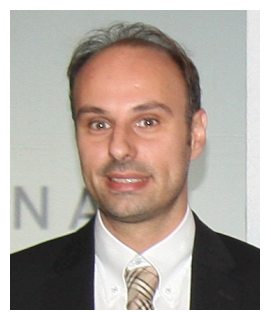

Ioannis Krikidis (S'03-M'07-SM'12) received the diploma in Computer Engineering from the Computer Engineering and Informatics Department (CEID) of the University of Patras, Greece, in 2000, and the M.Sc and Ph.D degrees from Ecole Nationale Supérieure des Télécommunications (ENST), Paris, France, in 2001 and 2005, respectively, all in electrical engineering. From 2006 to 2007 he worked, as a Post-Doctoral researcher, with ENST, Paris, France, and from 2007 to 2010 he was a Research Fellow in the School of Engineering and Electronics at the University of Edinburgh, Edinburgh, UK. He has held also research positions at the Department of Electrical Engineering, University of Notre Dame; the Department of Electrical and Computer Engineering, University of Maryland; the Interdisciplinary Centre for Security, Reliability and Trust, University of Luxembourg; and the Department of Electrical and Electronic Engineering, Niigata University, Japan. He is currently an Assistant Professor at the Department of Electrical and Computer Engineering, University of Cyprus, Nicosia, Cyprus. His current research interests include communication theory, wireless communications, cooperative networks, cognitive radio and secrecy communications.

Dr. Krikidis serves as an Associate Editor for IEEE Transactions on Communications, IEEE Transactions on Vehicular Technology, IEEE Wireless Communications Letters, and Wiley Transactions on Emerging Telecommunications Technologies. He was the Technical Program Co-Chair for the IEEE International Symposium on Signal Processing and Information Technology 2013. He received an IEEE Communications Letters and IEEE Wireless Communications Letters exemplary reviewer certificate in 2012. He was the recipient of the Research Award Young Researcher from the Research Promotion Foundation, Cyprus, in 2013. 